\title{
La Laurentide (1887-1928) : pionnière du papier journal au Canada
}

\section{Jorge Niosi}

Volume 29, numéro 3, décembre 1975

URI : https://id.erudit.org/iderudit/303464ar

DOI : https://doi.org/10.7202/303464ar

Aller au sommaire du numéro

Éditeur(s)

Institut d'histoire de l'Amérique française

ISSN

0035-2357 (imprimé)

1492-1383 (numérique)

Découvrir la revue

Citer cet article

Niosi, J. (1975). La Laurentide (1887-1928) : pionnière du papier journal au Canada. Revue d'histoire de l'Amérique française, 29(3), 375-415.

https://doi.org/10.7202/303464ar d'utilisation que vous pouvez consulter en ligne.

https://apropos.erudit.org/fr/usagers/politique-dutilisation/ 


\title{
LA LAURENTIDE (1887-1928): PIONNIÈRE DU PAPIER JOURNAL AU CANADA ${ }^{1}$
}

\author{
JORGE NIOSI \\ Département de sociologie \\ Université du Québec à Montréal
}

\begin{abstract}
«Par l'ampleur des investissements, par la modernité de l'équipement et par le taux de productivité, la fondation de la Laurentide Pulp (and) Paper marque la naissance de l'industrie moderne des pâtes et papiers dans le Québec. La Laurentide Paper est un phénomène qui appartient au XXe siècle québécois.» (J. Hamelin et Y. Roby: Histoire économique du Québec 1851-1896 (Fides, Montréal, 1971, 266-268).
\end{abstract}

Vers la fin du XIXe siècle les ressources forestières du nord-est des États-Unis s'épuisaient et la demande de papier journal augmentait. La tendance à la baisse des prix se renversa au début du $\mathrm{XX}$ siècle et les grandes chaînes de journaux des États-Unis intensifièrent leur pression afin d'obtenir du gouvernement américain l'abolition du tarif frappant l'entrée du papier journal ${ }^{2}$. L'embargo sur l'exportation de bois à pâte par les principales provinces canadiennes qui en produisaient (l'Ontario en 1900 et le Québec en 1910) et, finalement, l'inscription du papier journal sur la liste des produits d'entrée libre aux États-Unis en 1913, vont compléter les prérequis de la migration vers le Nord de l'industrie papetière américaine.

Et, pourtant, bien avant que ces conditions ne deviennent effectives, des capitalistes américains établissaient au Canada quelques entreprises papetières. Parmi elles la plus importante fut la Laurentide Co., la plus grosse firme canadienne de production de papier journal de 1898 à 1919 , et l'une des mieux administrées de l'Amérique du Nord. Ce caractère de pionnière était le résultat d'une seule circonstance: la Laurentide a été lancée par plusieurs des plus remarquables capitalistes forestiers des États-Unis et du Canada.

\footnotetext{
1 Ce texte a été élaboré dans le cadre d'une recherche collective sur l'histoire de la compagnie Consolidated-Bathurst Ltd, qui a mis ses archives à notre disposition. 2 L. Ellis, Print Paper Pendulum (N. Brunswick, U.S.A., 1948).
}

RHAF, vol. 29, no 3 (décembre 1975) 
Ces hommes étaient en mesure de prévoir le développement futur de l'industrie papetière nord-américaine grâce à leur connaissance du secteur: Albrecht Pagenstecher et Warren Curtis (fondateurs de la première usine de papier à base de pâte de bois en Amérique, et co-fondateurs de l'International Power and Paper en 1898), le général Russell Alger (grand entrepreneur forestier, commandant en chef de l'armée américaine, ministre de guerre sous McKinley et sénateur) et James MacLaren (l'un des principaux capitalistes forestiers et papetiers canadiens de la fin du XIXe siècle). L'usine a été ensuite prise en main par les plus puissants entrepreneurs canadiens du début du XXe siècle, les administrateurs du Canadien Pacifique et de la Banque de Montréal: William Van Horne, Richard B. Angus et James Ross, entre autres. Si le caractère exceptionnel de ses fondateurs explique la précocité de la Laurentide Co., la puissance financière de ses propriétaires lui donna sa taille. Ces deux traits de la Compagnie fondent l'intérêt historique de son étude.

Les orientations théoriques de notre recherche proviennent de différents courants de la sociologie et de l'histoire industrielles. Nous voulons mettre à l'épreuve plusieurs hypothèses concernant l'évolution de la corporation manufacturière moderne. Une première proposition qu'il nous intéresse de vérifier est celle de la séparation entre la propriété et le contrôle des entreprises par actions. Le débat fut ouvert par l'étude célèbre de Berle et Means (1932) ${ }^{3}$. Ils affirment que dans la corporation moderne la propriété des actions est dispersée entre les mains de milliers de petits propriétaires et que, dans ces conditions, le contrôle reste entre les mains de managers sans propriété. Cette théorie a été reprise par de nombreux auteurs, soit pour tenter de la confirmer avec de nouvelles données, soit pour la critiquer. Parmi les opposants (Perlo, 1958; Chevalier, 1969) on affirme que si le nombre d'actionnaires s'accroit sensiblement durant tout le XXe siècle, le contrôle des compagnies par les principaux actionnaires ne diminue pas pour autant et que les familles des fondateurs détiennent encore des positions dominantes dans bien des cas.

3 Les auteurs cités dans cette section sont notamment: A. Berle et G. Means, The Modern Corporation and Private Property (New York, 1932); V. Perlo, "People's capitalism and stock ownership», American Economic Review (1958), no 3; J.-M. Chevalier, La structure financière de l'industrie américaine (Paris, 1969); R. Hilferding, Le capital financier (1910) (Paris, 1970); P. Sweezy, The Present as History (New York, 1955); G. Kolko, The Triumph of Conservatism (Chicago, 1963); A. Chandler, Strategy and Structure (Cambridge, Mass., 1961). 
La théorie du capital financier de R. Hilferding (1910) est aussi mise à l'épreuve. Hilferding affirmait que les banques de placement (investment banks aux États-Unis et en Angleterre, banques d'affaires en France) tendent à contrôler les corporations et que ce contrôle est acquis grâce au financement des émissions d'actions et d'obligations. Contre cette position, tout au moins dans le contexte des États-Unis, se dressent, entre autres, P. Sweezy (1955) et G. Kolko (1963). Sweezy soutient que l'accroissement des liquidités des corporations leur a permis de se rendre indépendantes des banques de placement à compter de la première guerre mondiale.

Une deuxième hypothèse majeure est celle d'Alfred Chandler Jr (1961) sur l'évolution de la structure administrative des firmes. Chandler soutient que, jusqu'à la première guerre mondiale, la structure centralisée par départements était la seule forme organisationnelle des corporations et que, dans la période entre les deux guerres, quelques compagnies d'avant-garde (G.M., Sears-Roebuck, Du Pont, etc.) ont mis sur pied un nouveau type d'organisation: la structure divisionnelle décentralisée.

Au-delà de la mise à l'épreuve de théories, nous voulons aussi jeter un regard sur la composition de la grande bourgeoisie montréalaise, bien représentée à la Laurentide, en vue de la découverte et de l'énonciation de nouvelles hypothèses.

\section{I. - La Laurentide Pulp Co. (1887-1896)}

Située sur la rivière St-Maurice, à 28 milles au nord-ouest de Trois-Rivières, la région de Grand-Mère ne connut pas d'établissement fixe de population avant 1852. Cette année-là, la Couronne concédait de vastes territoires forestiers dans la région à plusieurs commerçants, dont George Baptist, de Trois-Rivières. Ce dernier, marchand de bois et propriétaire d'une usine de sciage aux Grès, fut un des principaux capitalistes forestiers du Québec au XIXe siècle. Il mourut en 1875 et plusieurs compagnies papetières durent s'adresser à ses successeurs pour l'achat de terres à bois. En 1856, fut créé le village de Ste-Flore, près de l'emplacement de la future ville de Grand-Mère, avec quelques dizaines d'habitants. La croissance de la population dans les trois décennies suivantes fut très lente. En 1880, un embranchement du chemin de fer arriva au village. En 1881, la paroisse de Ste-Flore comptait 1,268 habitants; le village lui-même avait quelque 250 habitants. 
Cette année-là, John Forman, immigrant écossais, commerçant à Montréal, visita le village et vit les possibilités de développement de la production de pâte et papier dans la région, grâce à la chute d'eau sur le St-Maurice et aux réserves forestières. Aussitôt il associa à son projet un groupe de capitalistes montréalais, obtint une exemption de taxes pour vingt-cinq ans du Conseil municipal du village (en mai 1882) et, en octobre, il incorporait la Canada Pulp Co. $L t d$. avec un capital autorisé de $\$ 50,000$. La Compagnie se fit accorder aussi la chute et l'île de Grand-Mère. Après quelques mois de construction, en septembre 1883, la Compagnie fit faillite par manque de fonds, sans même avoir pu terminer le canal hydroélectrique sur le St-Maurice. Deux mois plus tard, la Compagnie était liquidée et Forman acheta des terrains adjacents et chercha de nouveaux partenaires commerciaux pour relancer l'entreprise. Finalement, en 1887, il intéressa deux capitalistes américains et deux canadiens et repartit à nouveau ${ }^{4}$.

Qui étaient les fondateurs de la Laurentide que Forman associa à son projet? Albrecht Pagenstecher et Warren Curtis étaient les nouveaux partenaires américains; James MacLaren et John Doyle étaient les canadiens. Pagenstecher était né en 1839 à Osnabrück, Allemagne. Associé à son frère Rudolph et à son cousin Alberto, il bâtit le premier moulin en Amérique du Nord pour la production de pâte mécanique. Celle-ci avait été inventée en Allemagne en 1847 par Alfred Keller et Heinrich Vollter; leur brevet fut acheté par les Pagenstecher et leur associé Steinway. En 1866, ils introduisirent aux États-Unis les deux premiers appareils broyeurs (grinders) pour leur petite usine de Curtisville, Massachusetts. En mars 1867, l'usine des Pagenstecher vendit la première production de pâte mécanique de bois fabriquée en Amérique. Par la suite Pagenstecher bâtit un deuxième moulin à Luzerne, Warren County, N.-Y., et un autre à Palmer Falls, N.-Y. Ce dernier, construit en 1868-69, prit le nom de Hudson River Pulp and Paper Co. et devint la principale entreprise de Pagenstecher, ainsi que la première usine de pâte et papier aux États-Unis à employer de la machinerie faite dans le pays. Pagenstecher était associé à Miller, sénateur des États-Unis; le manager général de la firme était Warren Curtis, auquel nous reviendrons bientôt. En 1876, le même groupe fonda la Manufacturers Paper Co., entreprise papetière située dans l'État de New-York.

4 A. Désilets, La Grand'Mère. Coll. «Pages trifluviennes », vol. 10 (TroisRivières, 1933), 40-53; G. Carruthers, Paper in the making (Toronto, 1947), 599-610. 
En janvier 1898, Pagenstecher et Curtis participent à la monumentale fusion qui créa la plus grande corporation de pâtes et papiers au monde: L'International Power \& Paper Co. consolidant la Hudson River Co. Les deux associés devinrent alors membres du Conseil d'administration de l'I.P. \& P. Co. et plus tard de sa filiale spécialisée dans le papier kraft, la Continental Bag $\mathrm{Co}^{5}$. L'ingénieur Warren Curtis, né en 1836 aux Etats-Unis, était manager du Post Dispatch de St-Louis quand, en 1869, Pagenstecher l'engagea pour la Hudson River Pulp and Paper Co. En 1874, Curtis remodela la machine Fourdrinier pour la production de papier journal et employa avec succès son invention à la Hudson River Co. Curtis breveta sa découverte qui fut connue sous le nom de Broyeur Curtis (Curtis Grinder). À l'époque où Curtis s'intéresse à la Laurentide, sa réputation comme technicien des pâtes et papiers était faite et il travaillait à l'amélioration de la machine Fourdrinier. Il est mort en 1913.

James MacLaren est né en Écosse en 1818; sa famille s'était établie au Canada quand il était jeune. Il fut d'abord marchand de bois, puis acheta des limites forestières et mit sur pied une usine de sciage sur la Lièvre. Il fonda et présida jusqu'à sa mort la Banque d'Ottawa dont il était le principal actionnaire. Il fut vice-président du chemin de fer Ontario Central et fut, en 1885, l'un des fondateurs de la Canadian Copper Co. qui deviendra, en 1902, l'International Nickel Co. Dans le domaine papetier, il fonda la Buckingham Pulp Co., compagnie de fabrication de pâte, plus tard la James MacLaren Power and Paper Co. Il est mort en $1892^{6}$. Le dernier associé de la compagnie, John F. Doyle, marchand de Montréal, jouait un rôle mineur dans l'entreprise.

Les cinq associés obtinrent une charte fédérale en juin 1887. Le capital-actions autorisé était de $\$ 200,000$. divisé en 2,000 actions d'une valeur nominale de $\$ 100$. chacune. Pagenstecher apporta $\$ 34,000$. et reçut 340 actions, Curtis $\$ 24,000$. en échange de 240 actions, MacLaren acheta 100 actions pour $\$ 10,000$. et Doyle 20 actions pour $\$ 2,000$. Forman vendit ses propriétés à la compagnie pour $\$ 30,000$. en les échangeant contre 300 actions. Cinquante pour cent du capital-actions autorisé était donc souscrit par les fondateurs. Un autre $25 \%$ fut souscrit à la fondation même par des capitalistes

5 The Paper Mill and Wood News, vol. 49, no 33: 1 (le 14-8-1926); R.S. Kellog (1923): 19; L.T. Stevenson (1940): 22; PPMC (le 15-11-1913): 759.

6 Representative Canadians, A Cyclopedia of Canadian Biography (Toronto, 1888), 540-541; O. Main (1957). 
germano-américains, en particulier German F. Victor, de la German Savings Bank de New-York et de la Munick Reinsurance Co. et importateur de tissus, George Gudewill de la German Savings Bank, et le baron A.W. Von Kessler, noble allemand résidant aux ÉtatsUnis ${ }^{7}$.

À la fondation, le contrôle de la Compagnie restait donc entre les mains du Conseil d'administration, qui possédait $50 \%$ des actions, et d'un petit nombre d'associés: 14 actionnaires détenaient $85 \%$ des actions.

La nouvelle charte et les règlements internes stipulaient que la compagnie serait administrée par un Conseil de cinq membres élus annuellement par l'Assemblée des actionnaires. Lors de l'assemblée de fondation, Pagenstecher devint président, MacLaren vice-président, Curtis fut nommé trésorier, Forman secrétaire et Doyle vérificateur des comptes.

Les travaux d'excavation et de construction furent commencés immédiatement par 300 travailleurs sous la direction de Curtis. Quelques mois plus tard cependant les $\$ 200,000$. étaient dépensés et le Conseil d'administration se mit à la recherche de fonds supplémentaires. En août 1888 les actionnaires américains rejetaient une proposition du C.A. pour augmenter le capital-actions à $\$ 300,000$. et approuvèrent par contre un mois plus tard une émission d'obligations de $\$ 100,000$. pour financer la construction dont les coûts devenaient plus élevés que prévus. De toute évidence les bailleurs de fonds de la firme commençaient à considérer l'entreprise trop hasardeuse. Les obligations émises, portant un intérêt de 6\%, furent rapidement souscrites par les capitalistes germano-américains que Pagenstecher avait associés à la firme. Et pourtant, au début de la production (1888), la Compagnie était très endettée. La construction avait absorbé deux fois les coûts estimés. En juin 1889, le bilan évaluait les actifs totaux de la Compagnie à $\$ 399,00 \mathrm{C}$. et, dans le passif, apparaissaient de nombreux comptes à payer et des prêts bancaires.

À la fin de la période de construction, l'usine possédait quatorze meules (grindstones) de production de pâte mécanique avec une capacité de vingt-cinq tonnes par jour. En 1890, on commanda six meules et la machinerie complémentaire à Holyoke Machine Co. du Massachusetts et, en 1892, l'usine put augmenter sa capacité

7 Procès-verbaux du C.A., livre no 1: 13. 
de production à quarante-cinq tonnes de pâte par jour. En 1896 elle passa à 60 tonnes par jour: c'était la capacité maximum de l'usine avec les génératrices installées de 5,000 chevaux-vapeur.

Durant cette première étape, les coûts de production égalaient (et parfois dépassaient) les prix de vente. Un rapport présenté par deux membres du C.A. à l'assemblée annuelle des actionnaires, en octobre 1893, explique la situation:

Le coût moyen de la pâte l'an dernier fut de $\$ 9.80$, le prix moyen de vente de $\$ 13.63$, laissant un profit de $\$ 3.83$ par tonne soit pour une production de 11,700 tonnes un profit de $\$ 44,807$, de quoi payer l'intérêt des obligations, des prêts et du capital. Les deux premiers prennent $\$ 25,000$., laissant $\$ 20,000$. pour amortissement et dividendes. Il faut donc réduire le coût de production parce que le prix n'augmentera pas. ${ }^{8}$

En effet, depuis la fondation de la Laurentide Pulp le prix du papier journal et de sa principale matière première, la pâte mécanique, ne cessait de se détériorer. La tonne de papier journal, qui se vendait $\$ 68$. à New York, en 1890 , ne valait plus que $\$ 55$. en 1893 et \$36. de 1897 à $1901{ }^{9}$. Et le marché de la Laurentide était nord-américain. En octobre 1892, le marché européen avait commencé à être étudié par les administrateurs de la firme, et quelques milliers de tonnes furent vendues jusqu'en 1896 en Angleterre. La plupart des ventes se faisaient aux États-Unis malgré les tarifs, la concurrence locale et les fluctuations de prix.

L'expérience des deux dernières années a démontré l'irrégularité du marché de ce côté de l'Atlantique et nous en souffrons aujourd'hui, étant obligés de garder de vastes stocks de pâte pour laquelle il n'y a pas de demande à présent de nos clients habituels. ${ }^{10}$

Pour développer le marché européen, Forman est envoyé en Angleterre au début de 1894 et, en juin de cette année-là, devient agent de ventes de la Compagnie pour le Canada et l'Angleterre, moyennant une commission. Il conserva ce poste pendant un an et demi, démissionnant du C.A. en octobre 1894.

\footnotetext{
$8 \quad$ Procès-verbaux du C.A., livre no 2: 69.

9 Prix f.o.b. à l'usine pour la vente à New-York, en dollars courants. Les données sont celles de la Newsprint Association of Canada, citées par Bladen (1973), 178 .

10 Procès-verbaux du C.A., livre no $2: 69$.
} 
Les coûts de transport ont pu être réduits grâce à la construction, en 1891, par le Canadien Pacifique d'une voie ferrée jusqu'à l'usine. La Laurentide se trouvait ainsi reliée au plus grand réseau ferroviaire canadien. Quant à l'approvisionnement en bois, la politique de la Compagnie fut d'obtenir la matière première de ses propres limites forestières. Ainsi, en 1890, la Laurentide acheta 324 milles carrés sur le St-Maurice pour la somme de $\$ 30,000$., mais dut revendre 100 milles carrés en 1895 à la Hudson River Co. pour $\$ 20,000$. à cause du besoin pressant de capital de la Compagnie. Le bilan net de la période fut alors très positif au point de vue de l'équipement et des réserves forestières, de l'approvisionnement hydroélectrique et du transport. L'instabilité des marchés et des prix menaçait cependant la rentabilité de la firme.

La capitalisation de la Compagnie n'était pas facile dans ces conditions. En juillet 1889 , on mit en vente $\$ 100,000$. supplémentaires d'actions accordant une première option aux actionnaires en proportion de leur avoir, sous réserve de vente au public en cas de refus. En juillet 1892, $\$ 120,000$. d'obligations étaient vendues, et l'on contracta quelques dettes envers des banques montréalaises. On constate un souci évident des principaux actionnaires (administrateurs ou non) de restreindre l'émission d'actions et ceci avec un triple but: d'abord, de s'assurer une rente sur les mises de fonds, rente assurée seulement par les obligations; ensuite, de se prévenir contre une éventuelle faillite; finalement, de conserver le contrôle de la Compagnie qui leur aurait échappé si des actions avaient été vendues massivement sur le marché boursier. Ainsi, en 1888, le capital-actions représentait $84 \%$ du passif, mais en 1896 il n'en représentait que $37 \%$, alors que les obligations en représentaient déjà $27 \%$. Il n'y eut pas de dividendes pendant ces dix premières années de vie de la Laurentide.

Au sein de la Compagnie, il n'y avait qu'un bureau central: administrateurs et officiers étaient les mêmes personnes. Les administrateurs comprenaient le président, poste occupé par Pagenstecher pendant toute la période (de juillet 1887 à février 1897), le viceprésident, poste assumé successivement par James MacLaren (de juillet 1887 à août 1890), F. W. Evans (de 1890 à octobre 1894) et W. Curtis (de 1894 à février 1897). Curtis fut le trésorier de 1887 à 1897 sans interruption; Forman en fut le secrétaire de 1887 à 1894; à partir de cette date, John Riley, ancien surintendant du moulin, le remplace. Doyle devient le vérificateur des comptes de la Compagnie (et membre du C.A.) de 1887 à 1890 , date où le poste 
et son titulaire furent séparés du Conseil. Walter Drake de Montréal en sera le cinquième administrateur. Une fois Forman disparu de la direction de la Compagnie, le contrôle de la Laurentide resta entre les mains des entrepreneurs américains. Evans, Riley et Drake n'avaient que quelques actions chacun et ils ne jouèrent qu'un rôle secondaire au C.A. En décembre 1892, le nombre des membres du C.A. passa de cinq à sept. Plusieurs administrateurs se sont succédé à ces nouveaux postes dont les plus forts actionnaires du groupe Pagenstecher-Curtis: C. E. Gudewill, George Victor, H. Dewitt et Peter Mc Carthy. Parmi les nouveaux administrateurs, un seul Canadien, F. Farmer, décrit par A. Désilets comme "agent de $\mathbf{M}$. Curtis à Trois-Rivières ${ }^{11}$. Il s'agissait sans doute pour les actionnaires et obligataires américains de conserver un contrôle étroit sur la Compagnie dont le succès était loin d'être assuré.

La structure administrative de la Compagnie était simple: le Conseil d'administration concentrait tous les pouvoirs. Quelques comités spéciaux furent formés à l'occasion: le comité de construction, de 1887 à 1891, composé de Curtis et Forman; le comité de négociation avec le C.P.R. en 1891, un autre pour l'émission d'obligations. En 1892, on créa un comité exécutif (Evans, Drake et Forman) et un comité financier (Pagenstecher, Victor et Curtis). Le premier s'occupait de la marche quotidienne de la firme, le deuxième des grandes décisions financières, telles que l'emprunt et l'investissement. Ces deux comités ne durèrent qu'un an, probablement à cause de la présence des principaux actionnaires au C.A. Cette structure extrêmement centralisée subit des changements dès la fin de 1893. En effet, le rapport minoritaire de Drake et Evans, en octobre de cette année-là, proposait d'adopter une structure départementale, du type récemment adopté dans les corporations industrielles des États-Unis:

En conclusion des points antérieurs nous tenons à faire les suggestions suivantes: que le management des affaires de la Compagnie soit divisé en trois départements de la manière qui suit.

Toutes les questions liées à la manufacture à Grand-Mère seraient sous le contrôle d'un Manager résidant à cet endroit; toutes les questions liées à l'achat de ressources pour l'entrepôt et la vente de produits de la Compagnie seraient pris en charge par un Agent ou Représentant résidant à Montréal; un Bureau sera entretenu à Montréal pour tenir les livres de la

11 A. Désilets, op. cit., 58. 
compagnie. Ce bureau étant dirigé par un administrateur qui, à notre avis, devrait être le secrétaire.

La personne en charge de chacun de ces départements serait sous le contrôle immédiat du Conseil d'Administration, et serait responsable entièrement de son département. ${ }^{12}$

L'organisation proposée fut adoptée. Le siège social était déjà établi à Montréal depuis décembre 1892. Dix mois plus tard, Forman était nommé agent de ventes de la compagnie et séparé du C.A. Curtis conserva la direction technique de la manufacture.

En guise de conclusion, on peut dire que la Laurentide, pionnière dans le processus de migration vers le nord de l'industrie américaine de pâtes et papiers, connut, pendant ses dix premières années, toutes les difficultés de la manufacture moderne à ses débuts. Audelà de la grosse erreur d'estimation des capitaux requis pour l'installation de l'usine (erreur qui eut pour effet de mettre la Compagnie en situation financière précaire), l'état du marché nordaméricain du papier et le tarif protecteur des États-Unis n'aidèrent pas non plus. Après un bref boom de quelques mois (fin 1887 à juillet 1890) les prix de la pâte mécanique tombèrent régulièrement. Avant le boom, la pâte se vendait $\$ 80$. la tonne courte; durant la brève flambée elle augmentera à $\$ 120$. et $\$ 200$. la tonne, pour ensuite retomber à $\$ 12$. la tonne, à l'époque des consolidations papetières aux États-Unis (1896-1898). Dans ce cycle de prix à la baisse, de surinvestissement et de concurrence sans restriction, la Laurentide était aussi défavorisée par le tarif américain frappant l'importation de pâte. En 1883, le Mongrel Tariff avait supprimé toute protection douanière sur le bois à pâte et fixé à $15 \%$ le tarif douanier du papier journal. En 1890, le McKinley Tariff maintient ce taux de $15 \%$ pour le papier journal et porte à $\$ 2.50$ la tonne le prix de la pâte mécanique, ce qui signifie une augmentation de la protection douanière. Avant cette modification, il aurait fallu payer pour une tonne de pâte d'un prix moyen de $\$ 12$. un tarif douanier de $\$ 1.20$. Les éditeurs américains ne s'objectaient pas encore au tarif douanier: les prix étant bas, le marché de pâte et papier était à la hausse.

À l'intérieur de la Compagnie, propriété et contrôle ne sont pas séparés; les principaux actionnaires se retrouvent au Conseil d'administration. D'autre part, la firme présente des caractéristiques

12 Procès-verbaux du C.A., livre no 2: 71. 
de l'industrialisation des pays périphériques: la technologie, le "know how», le financement et l'organisation viennent des États-Unis. Les Canadiens n'y jouent qu'un rôle subordonné, de junior partners.

\section{II. - La Laurentide Pulp (II: 1896-1903)}

En mars 1896 la Compagnie change de mains. Le général Russell, A. Alger, alors commandant en chef de l'armée américaine, achète 2,003 actions $(2 / 3$ du capital-actions) pour $\$ 150,000$. soit $\$ 75$. l'action. Il acquiert ainsi le contrôle total de l'entreprise. Il vend aussitôt 500 actions à sir William Van Horne et 300 autres à Richard B. Angus. Ces trois actionnaires formèrent le noyau du groupe qui contrôla la Laurentide pendant cette deuxième étape.

Pour quelles raisons Pagenstecher et Curtis ont-ils vendu leurs actions dans la Laurentide avec une perte de capital, entraînant avec eux le groupe germano-américain? Nous avons vu que l'entreprise n'était pas rentable pour les actionnaires et que les prévisions ne permettaient pas d'entrevoir une amélioration des prix de la pâte. En outre Curtis et Pagenstecher étaient à la tête de plusieurs compagnies papetières aux États-Unis et ils préparaient la fusion qui donna lieu à la fondation de l'International Paper en janvier 1898. D'autre part, le développement de la Hudson River entre 1895 et 1898 demanda beaucoup d'efforts aux deux associés; cette entreprise était d'ailleurs la plus importante parmi celles qui ont formé l'International Paper en 1898. Il était donc normal que Pagenstecher et Curtis consacrent leurs ressources et leurs énergies à la plus grosse, la plus rentable et la plus dynamique de leurs entreprises.

Les acheteurs de la Laurentide formaient un groupe important de millionnaires des États-Unis et du Canada. Le général Alger était né à Lafayette, Medina, Ohio en 1836. Il travailla d'abord comme journalier dans une ferme, fut successivement maître d'école, avocat au barreau (1859) et officier de l'armée nordiste en 1861. À la fin de la Guerre de sécession, il s'engagea dans des affaires forestières. Bientôt il eut des intérêts prédominants dans les firmes suivantes: Alger, Smith and Co., (possédant de grandes réserves forestières et des scieries au Michigan et à la Baie Georgienne au Canada), Detroit and Rio Grande Cattle Co. of New Mexico, Manistee Railway Co., Marais Lumber Co., State Savings Bank of Detroit, Union Lumber Company of Cleveland et United States Express Co. En 1885-86, il devint gouverneur de l'État du Michigan et en 1888 se porta candidat à la présidence de la Convention nationale républicaine. Par 
la suite il devint commandant en chef de l'armée américaine, poste qu'il occupait quand il acheta la Laurentide, puis promu ministre de Guerre sous McKinley de 1897 à $1899^{13}$.

Sir William Cornelius Van Horne est une figure trop connue pour que nous insistions sur sa biographie. Il suffira de souligner qu'après son passage à la présidence du Canadien Pacifique il acheta, avec le général Alger, deux compagnies papetières: La Laurentide Pulp et la Glenn Falls Co. du Nouveau-Brunswick. Son amitié avec Alger, et plus tard aussi avec Taft, lui offrit la possibilité de construire plusieurs chemins de fer en Amérique centrale et aux Antilles où l'influence américaine augmenta à la suite de la guerre hispanoaméricaine ${ }^{14}$.

Richard Bladworth Angus naît en 1831 à Bathgate, Écosse. En 1857, il entre comme employé à la Banque de Montréal. En 1869, il en devient le gérant général, poste qu'il conserve pendant deux ans. En 1881, nommé administrateur et membre du comité exécutif du C.P.R., Angus s'est entre-temps associé à George Stephen (qui laissait la présidence de la Banque de Montréal pour assumer celle du Canadien Pacifique) et à Donald Smith dans le St. Paul, Minneapolis and Manitoba Railway, ainsi que dans des entreprises textiles comme la Lamas Mills et la Quebec Worsted Co. En 1883 Angus assume la vice-présidence du C.P.R. et en 1887 il retourne au Comité exécutif du Conseil d'administration du Canadien Pacifique jusqu'à sa mort en 1922. En 1891 Angus revient au C.A. de la Banque de Montréal, est élu administrateur de la Laurentide en 1898 , et siège au C.A. de plusieurs firmes industrielles, commerciales et ferroviaires.

Les principaux capitalistes attirés par Van Horne et Angus à la Laurentide se nomment James Ross, Charles R. Hosmer et William Mackenzie. James Ross né en Écosse en 1848, fit des études d'ingénieur civil en Grande-Bretagne avant d'aller aux États-Unis en 1868 et d'immigrer au Canada en 1874. En 1883, il fut embauché par le C.P.R. pour superviser la construction de la voie à l'ouest de Winnipeg. Plus tard il s'occupa de la construction de chemins de fer, tramways et usines hydro-électriques au Canada, en Angle-

13 Who's Who in America (1899-1900 et 1903-1905); Directory of Directors of the City of New York (the Audit Co. of New York, 1898); The Financial Red Book of America (1905).

14 Walter Vaughan, The Life and Work of Sir William Van Horne (New York, 1920); Who's Who in America (1910); Who's Who in Canada (1910-1911). 
terre, au Mexique et dans les Caraïbes et devint président de la Dominion Coal Co., de la compagnie Dominion Bridge, et du chemin de fer de St-John, administrateur de la Banque de Montréal de 1899 à 1913, de la Canadian General Electric et d'autres entreprises. Il eut un fils, John K.L. Ross, qui lui succéda en 1915 au Conseil d'administration de la Laurentide; James Ross en fit partie de 1897 à 1915 .

Charles R. Hosmer né au Québec en 1851, fut nommé en 1884, directeur du service de télégraphie du C.P.R. dont il devint administrateur en 1899. En 1908, il devint aussi administrateur de la Banque de Montréal. Il siégeait en outre au C.A. de nombreuses entreprises importantes de Montréal, dont Sun Life Assurance, Montreal Light Heat and Power, le Royal Trust, Dominion Express et autres.

Sir William Mackenzie avait commencé sa carrière en affaires comme sous-traitant du C.P.R. En 1896, associé à Donald Mann, il acheta son premier chemin de fer, le Lake Manitoba Railway and Canal, qui servit de base au Canadian Northern. Il contrôlait aussi plusieurs compagnies hydro-électriques du Niagara et de Toronto. Premier président de la Brazilian Traction Light and Power, il siège de même au C.A. de la Laurentide de 1897 à 1902.

De son côté, le général Alger associa son fils Russell Alger $\mathrm{Jr}$ et ses trois filles à la Compagnie. Les quatre se retrouvent parmi les principaux actionnaires et, de plus, Alger Jr fut trésorier de 1897 à 1900 et manager de 1900 à 1901. Le général Samuel Thomas de New-York et le banquier George B. Hopkins furent associés aussi à l'entreprise par le général Alger lors de l'émission d'actions de septembre 1899. En mars de cette année-là les principaux actionnaires étaient les suivants: le général Alger possédait 2,580 actions, Van Horne en avait 1,390, Mackenzie 1,030, Angus, 1,000. Six autres actionnaires en possédaient 2,600 en tout. En somme dix personnes possédaient trois quarts du capital-actions et le C.A. en détenait plus de $50 \%{ }^{15}$.

Peu après l'arrivée du groupe Alger-Van Horne, la Laurentide commença à diversifier sa production et à l'augmenter. On commanda trois machines Fourdrinier (de 90, 110 et 120 pouces) et, au printemps de 1899, la capacité installée était de 120 tonnes de pâte mécanique par jour, 70 tonnes de pâte au bisulphite, 40 tonnes de papier journal et 35 tonnes de carton-pâte. En 1900, on ajoutait une qua-

15 Procès-verbaux du C.A., livre no $2: 252$. 
trième machine Fourdrinier de 120 pouces, pour augmenter la capacité de production de papier journal à 75 tonnes. Avec ces développements, la Laurentide devenait la plus grosse usine canadienne de pâtes et papiers. La capacité hydro-électrique fut augmentée parallèlement.

Nous n'avons pas de renseignements sur les coûts de production de la Laurentide Pulp dans cette étape d'expansion. Toutefois, on sait que les prix de la pâte et du papier en Amérique du Nord sont encore tombés durant cette période. Le papier journal, qui se vendait $\$ 45$. la tonne en 1896 , ne valait plus que \$36. entre 1897 et 1901 ; en 1902 , il augmenta à $\$ 38$. et, en 1903 , à $\$ 41$. (ce sont les prix à New-York). Au Canada, les prix du papier et de la pâte suivirent le même cours qu'aux États-Unis, c'est-à-dire à la baisse. Pour faire face à cet état du marché, la Laurentide forme, en février 1900, un gentlemen agreement, un accord portant sur les prix de vente du papier journal au Dominion et à Terre-Neuve, avec vingt-cinq autres entreprises canadiennes ${ }^{16}$. Ce fut la Paper Makers' Association of Canada, qui fixa d'abord les prix à $\$ 50$. la tonne de papier journal en rouleau et $\$ 55$. en feuille, ce qui impliquait des augmentations de 25\% par rapport au prix antérieur. En février 1901, les prix furent de nouveau augmentés à $\$ 55$. et $\$ 60$. la tonne, selon le type. La réponse des propriétaires d'imprimeries et de journaux se fit bientôt sentir. En avril 1901, ils réussissaient à faire mettre sur pied une Commission Royale d'enquête sur l'accord. Cette enquệte, dirigée par le juge Henri-Thomas Taschereau, de la Cour Suprême du Canada, permit de connaître les termes de l'agreement. En mai 1901, sous la pression de la Commission d'enquête et de la Canadian Press Association, le prix du papier fut réduit à $\$ 47.50$ la tonne. En novembre 1901, la Commission remettait son rapport, concluant à l'existence de l'action illégale et, en février 1902, le gouvernement fédéral décidait de réduire le tarif protégeant le papier

16 Ces entreprises «étant pratiquement tous les producteurs de papier journal et d'imprimerie au Canada", selon le rapport du juge Henri-Thomas Taschereau en 1902, étaient par ordre alphabétique: W. Barber and Brothers, Alex Buntin and Sons, The Canada Paper Co. Ltd, The Dominion Paper Co. Ltd, The E.B. Eddy Co. Ltd, J. Fisher and Sons, J. Ford and Co., S.A. Lazier and Sons, The Lincoln Paper Mills Co. Ltd, Alex McArthur and Co., Miller Brothers and Co., The Northumberland Paper and Electric Co., The Ottawa Paper Co., The Riordon Paper Mills Co., The Royal Paper Mills Co., Reid Craig and Co., the Rolland Paper Co., J. Stutt and Son, The Ste Croix Pulp and Paper Co., The Toronto Paper Manufacturing Co., The Trent River Co., C.W. Thompson, The Thompson Paper Co., Taylor Brothers, J.C. Wilson and Co. (Rapport Taschereau, Ottawa, Imprimeur du Roy, 1902), 11. 
journal de $25 \%$ à $15 \%$ ad valorem. Peu de temps après l'Association de Manufacturiers de Papier du Canada est dissoute. Le retrait de la Laurentide s'est produit en juillet 1902 par décision unanime de son C.A. À la réunion du 28 juillet le président Van Horne annonçait aux administrateurs:

qu'il a découvert que cet accord avait été violé par la Compagnie Eddy, qui avait passé un contrat à un prix plus bas que celui de l'Association avec la Globe Printing Co., un contrat que notre compagnie avait refusé de signer à cause de l'agreement adopté pour maintenir le statu quo. Dans ces circonstances, le président recommande que la Laurentide Pulp s'occupe de ses propres affaires et se sépare de l'Association Canadienne de Manufacturiers de Papiers, et cette recommandation fut approuvée. ${ }^{17}$

L'incident est remarquable en tant qu'exemple des premières tentatives de concentration dans l'industrie canadienne de pâtes et papiers. Ces tentatives se résumaient à des gentlemen agreements entre beaucoup de concurrents. L'éparpillement de la production empêchait ces accords de réussir. Il faudra attendre la Première Guerre mondiale pour voir la deuxième étape de la concentration dans le secteur, centrée cette fois-ci autour des compagnies de ventes communes.

L'entrée des nouveaux propriétaires marqua une étape de capitalisation très rapide pour financer l'expansion. En mai 1897, l'Assemblée des Actionnaires approuvait une augmentation du capital-actions de $\$ 300,000$. à 900,000 .; en mars 1898 il est porté à $\$ 1,200,000$. et, en juillet 1899, à $\$ 1,600,000$. En même temps, on émet des obligations pour $\$ 1,200,000$. Le rapport du capital-actions au passif total se maintient à environ $35 \%$ durant toute la période; l'explication se trouve, à notre avis, dans la puissance financière du groupe qui détenait le contrôle de l'entreprise. A chaque nouvelle émission d'actions, il était en mesure d'acheter une partie considérable des certificats, tout en respectant les proportions respectives de chacun.

À partir de l'expansion de 1897, la Laurentide devint rentable pour ses actionnaires. Elle paya ponctuellement l'intérêt des obligations et distribua des dividendes de $3 \%$ en 1898 et en 1899 , de $7 \%$ en 1900 , et de $8 \%$ de 1901 à 1903 . L'intégration verticale des opérations permit à l'entreprise d'atteindre un seuil de rentabilité confortable. 
La structure administrative de la Compagnie fut modifiée pour distinguer davantage les fonctions d'entrepreneur (décisions d'investissement, de diversification, d'expansion, d'émission d'actions et d'obligations) des simples tâches d'exécution quotidienne routinières. Ainsi, en juin 1900, on décida de séparer du Conseil d'administration les tâches de secrétaire, trésorier, comptable en chef et directeur général. Les titulaires de ces postes nommés par le C.A. seraient responsables auprès du Conseil de leurs activités respectives. Aussi, en septembre 1902, on modifia les règlements internes pour permettre la nomination par le C.A. d'un ou de plusieurs viceprésidents non membres du Conseil qui s'occuperaient des affaires exécutives internes de la Compagnie; on s'acheminait ainsi vers la formation d'un Conseil d'officiers, même si le nom n'a pas été repris. $\mathrm{Au}$ poste de vice-président et directeur général, on nomma George Chahoon Jr, un Américain de New-York, né en 1872 et qui arriva à la Laurentide comme employé, sans propriété d'actions. D'autre part, la firme avait déjà un département d'opérations forestières, un autre de production de pâte, un troisième de production de papier et un quatrième de ventes. Finalement, en novembre 1897, l'Assemblée des actionnaires créa le Comité exécutif du Conseil d'administration sous forme permanente. Ce comité serait élu par et parmi les membres du C.A. formé de trois membres. Il posséderait et exercerait, par vote unanime de ses membres, tous les pouvoirs du C.A., quand le Conseil ne serait pas en réunion. Le Comité exécutif du C.A. se composait de Van Horne, Angus et Mc Gibbon, en 1897-98; de Van Horne, Angus et Alger Jr, de 1898 à 1900, et de Van Horne, Cassils et Angus, de 1900 à 1902. Ce comité exécutif avait un rôle à la fois financier (prise de décisions clefs et exécutif proprement dit).

Le contrôle de la Compagnie resta entre les mains des principaux actionnaires, membres du C.A. et de son Comité exécutif. À l'exception du général Alger et de son fils, les autres administrateurs et grands actionnaires ont fait leur carrière et leur fortune dans les chemins de fer (Van Horne, Mackenzie, Angus, Hosmer et Ross), particulièrement dans le Canadien Pacifique et le Canadian Northern, et dans la Banque de Montréal. Le Royal Trust qui émettait et enregistrait les transferts d'actions, était une société de fiducie fondée par les administrateurs de la Banque de Montréal.

Des directeurs de la Laurentide (Angus et Hosmer) siégeaient au Conseil d'administration. La compagnie se trouva ainsi contrôlée et appropriée par la grande bourgeoisie montréalaise, dont les sources 
de capital provenaient des grandes compagnies de chemins de fer et de la Banque de Montréal. On ne peut cependant pas parler de capital financier (au moins au sens de Hilferding) puisqu'il y manquait un élément essentiel: la banque d'investissement industriel prenant une partie centrale des actions (donc le contrôle) et les obligations. La Laurentide faisait appel à la Banque de Montréal pour des emprunts à court terme qui étaient remboursés en quelques mois.

\section{III. - La Laurentide Paper Co. (1903-1911)}

Afin de pouvoir diversifier ses activités, la Laurentide Pulp avait demandé des lettres patentes supplémentaires en juin 1903 à Ottawa; le gouvernement fédéral les accorda aussitôt sous l'égide de la nouvelle loi de compagnies de 1903. Les objectifs de la nouvelle charte étaient, premièrement, d'augmenter le capital-actions de 1.6 millions de dollars à 2.8 millions par l'émission de 12,000 actions privilégiées d'une valeur au pair de $\$ 100$. et donnant droit à des dividendes fixes de $7 \%$ par année. Cette capitalisation avait pour but premier l'élargissement de la production de papier pour transformer entièrement la pâte fabriquée à l'usine. Deuxièmement, il s'agissait d'étendre les pouvoirs légaux de la Compagnie pour lui permettre d'exercer toutes les activités manufacturières, forestières, de transport et d'utilités publiques, reliées et à la production du papier et à l'administration des services municipaux. Enfin, il s'agissait aussi de changer le nom de la compagnie en Laurentide Paper Co., plus conforme aux nouvelles normes. L'extension des pouvoirs demandée par la Compagnie résultait, d'une part, de la diversification de ses opérations manufacturières et d'autre part, elle répondait aussi à la croissance de la ville de Grand-Mère et à la possibilité entrevue par les administrateurs de la Laurentide de faire intervenir l'entreprise dans le secteur municipal: hôtellerie, distribution d'électricité, entreposage, etc.; finalement, la Compagnie prévoyait déjà la création de filiales, qui ne furent créées qu'au début de la période suivante.

La direction de la firme subit peu de changements dans cette étape; Van Horne, Angus, Hosmer et Ross restèrent au Conseil d'administration pendant toute la période. Le général Alger, par contre, se départit lentement de ses actions à partir de 1902, pour vendre les dernières en janvier 1906, date où il démissionna de son poste d'administrateur de la Laurentide; avec lui, son fils et ses filles vendaient leurs actions. Alger, sénateur des États-Unis en 1903, meurt en 1907. 
Parmi les nouveaux administrateurs, il y eut Edwin Hanson, courtier en valeurs mobilières, membre des maisons Hanson Bros et Hanson and Ferguson; la première de ces maisons, spécialisée en obligations, était l'une des plus prestigieuses du Canada. On la retrouve d'ailleurs dans la liste des principaux actionnaires.

Le managing vice-president, George Chahoon $\mathrm{Jr}$, remplaça le général Alger au Conseil d'administration de la Laurentide en janvier 1906. Peu de temps après, il commençait à acheter des actions de la Laurentide et, en 1911, si l'on additionne les actions qu'il possédait et celles de sa femme, il se situait parmi les plus forts actionnaires, avec 1,500 actions, soit $4.4 \%$ de toutes les actions souscrites. Lord Strathcona (D. Smith) était le dernier des grands actionnaires de la Laurentide. Il serait inutile de répéter les données biographiques du capitaliste le plus connu de l'époque de la Confédération. A sa mort, à 94 ans, en 1914, il avait été gouverneur de la Compagnie de la Baie d'Hudson, administrateur de la Banque de Montréal pendant quarante-deux ans (dont dix-huit à la présidence et cinq à la viceprésidence), administrateur du Canadien Pacifique et l'un des principaux actionnaires et administrateur de la Dominion Steel Corporation, de l'Anglo-Persian Oil Co. et nombre d'autres compagnies, député fédéral et Haut-Commissaire du Canada en Angleterre à partir de 1897.

La proportion des actions contrôlée par le C.A., de 19\%, augmente à $24 \%$, si nous y ajoutons les actions détenues par les membres proches de la famille des administrateurs, ainsi que les maisons de courtage qu'ils contrôlent. La Compagnie reste dirigée par la haute bourgeoisie montréalaise, celle de la Banque de Montréal et du Canadien Pacifique. Un phénomène nouveau, et qui prit de l'ampleur plus tard, fut la présence de maisons de courtage parmi les principaux actionnaires. Ces maisons gardent les actions pendant des années, ce qui nous empêche d'expliquer le fait qu'elles conservent des actions par des retards dans la distribution au public d'actions nouvellement émises; en outre, plusieurs de ces maisons se portent acquéreurs d'actions pour leur propre compte pendant de longues périodes. Puisque les fonctions fiduciaires leur étaient interdites, une seule explication semble prévaloir: les maisons de courtage achetaient ces actions avec leurs fonds et en étaient les propriétaires bénéficiaires. À l'appui de cette explication nous devons ajouter que, partout en Amérique du Nord, les grandes maisons de courtage (au Canada des sociétés comme Wood, Gundy and Co., A.E. Ames and Co., Ch. Meredith and Co. et d'autres) agissaient comme de véritables banques d'investisse- 
ment. Cependant, à l'exception d'Edwin Hanson, aucun courtier ne se trouvait au Conseil d'administration de la Laurentide. D'ailleurs, une seule banque à charte figure sur la liste des actionnaires importants en 1911 et elle l'a fait pour une période trop courte pour qu'on y voit une possibilité d'influencer les décisions de l'entreprise.

L'expansion que le C.A. promouvait en 1902-3 s'est réalisée dans les deux années qui suivirent la réception de la nouvelle charte. En 1911, la Laurentide produisait 170 tonnes de pâte mécanique par jour, 80 tonnes de pâte au bisulphite, 170 tonnes de papier journal et 40 tonnes de carton-pâte par jour. À partir de l'élargissement, la Laurentide ne vendait que du papier journal et, marginalement, du carton et un excédent de quelque 40 tonnes par jour de pâte au bisulphite. L'augmentation de la capacité de production fut réussie grâce à la mise en opération de plusieurs nouvelles machines: deux Fourdriniers (de 100 à 124 pouces) et une machine à cylindres de 120 pouces. La Compagnie possédait d'ailleurs treize broyeurs, trois digesteurs et quatorze machines «sèches" (Wet Machines).

Les prix du papier journal sont cependant restés stagnants, avec une légère hausse à la fin de la période: de \$40. la tonne (1904-7) ils passèrent à $\$ 42$. en 1908 et à $\$ 45$. entre 1909 et 1911 . Plusieurs développements économiques et politiques ont modifié le prix du papier journal: d'abord, la croissance permanente de la demande américaine face à une production interne qui se trouvait bloquée par l'épuisement des ressources naturelles; d'autre part, le Québec et l'Ontario imposaient des mesures pour obliger l'industrie américaine à migrer vers le nord. En 1900, l'Ontario avait émis un embargo sur l'exportation de bois à pâte coupé sur les terres de la Couronne. En 1901, le gouvernement du Québec créait un droit de coupe de 65 cents la corde pour le bois à pâte exporté, coupé dans les forêts de la Couronne. En 1910, le Québec imposait à son tour l'embargo sur le bois à pâte exporté, coupé dans les forêts de la Couronne. Durant cette étape, les producteurs américains de papier commencèrent à migrer vers le nord, mais leur installation prenant plusieurs années à se matérialiser, les compagnies déjà installées au Canada, comme la Laurentide, ont profité de cette conjoncture de transition pour réaliser des bénéfices supplémentaires. Aux États-Unis, le papier journal connut une réduction du tarif en 1909 , de $\$ 6.00$ à $\$ 3.75$ la tonne (plus $\$ 2.00$ la tonne si le papier journal était produit avec du bois en provenance des terres de la Couronne soumises à un embargo). En 1911, le traité de Réciprocité mit le papier journal sur la liste de libre-entrée à l'exception d'un impôt de $\$ 2.00$ la tonne sur le papier produit avec 
le bois sous embargo. Cependant, la défaite du gouvernement fédéral libéral, dirigé par sir Wilfrid Laurier, aux élections de septembre 1911, retarda de deux ans l'ouverture complète du marché américain pour les pâtes et papiers produits au Canada. Finalement, ce fut la pression des maisons d'imprimerie et des grands journaux américains qui finit par décider du sort de la bataille; en 1913, l'Underwood Act des États-Unis déclarait libre l'importation de papier journal dont le prix n'excédait pas $\$ 45$. la tonne (plus tard cette limite supérieure fut changée). Entre temps, l'embargo québécois imposé par le gouvernement libéral de sir Lomer Gouin en 1910 faisait son effet et l'industrie de la pâte et du papier se trouvait en pleine expansion en 1911.

En octobre 1903, le C.A. autorisait la Banque de Montréal à émettre les 12,000 actions privilégiées que l'assemblée des actionnaires avait votées en juin. L'émission, qui fut en partie achetée par les grands détenteurs d'actions ordinaires (le général Alger, Van Horne, Angus, Ross, lord Strathcona et sir W. Mackenzie en achetèrent 5.000 actions), avait comme objectif de constituer un fonds de roulement propre à l'entreprise, lui évitant ainsi de contracter des prêts bancaires à court terme. Pendant toute la période, la Compagnie racheta les obligations qu'elle avait émises en 1900; en 1911 il n'en restait que les trois quarts sur le marché. Comme conséquence de la politique de réduire les dettes fixes et surtout à partir de l'émission de $\$ 1,000,000$. d'actions ordinaires en 1909 , la part du capital-actions dans le passif augmenta de $35 \%$ en 1903 à $55 \%$ en $1911^{18}$. L'émission décidée en septembre 1909 ne fut pas écoulée totalement et elle porta le capital-actions à $\$ 3,400,000$. puisque 4,000 actions sont restées invendues.

Les profits de la Compagnie se sont maintenus stables de 1902 à 1909 , entre $8.5 \%$ et $9.5 \%$ par année. En 1910 , grâce à l'embargo du bois à pâte, la rentabilité est montée à $13.5 \%$ pour atteindre $15.6 \%$ en 1911. Les dividendes cependant sont restés à $7 \%$ pour les actions privilégiées et à $7 \%$ en $1904,6 \%$ de 1905 à $1907,7 \%$ en $1908-1910$ et $8 \%$ en 1911 pour les actions ordinaires. Cette politique de rétention de profits avait comme objectif la capitalisation rapide de l'entreprise sur ses fonds propres. Une partie de ces fonds fut utilisée pour acheter de nouvelles limites forestières; en 1904 la Laurentide possédait 1,650 milles carrés et 2,200 milles carrés en 1911. La ruée des compagnies papetières américaines sur la forêt canadienne fit augmenter

18 Il s'agit dans chaque cas des données des bilans annuels, au 30 juin de chaque année. 
le prix des terres à bois. Dans ces conditions (capitalisation par rétention des bénéfices, augmentation de la valeur des actifs), le C.A. décidait en 1911 une nouvelle réorganisation qui fera l'objet de la première partie du point suivant.

La structure administrative ne connut pas de changements importants. Le C.A. continua à exercer un contrôle complet sur l'entreprise. Ses membres étaient recrutés parmi les principaux actionnaires individuels: Van Horne, Angus, Alger (jusqu'à 1906 et ensuite George Chahoon Jr), Hosmer, Ross, Hanson et Charles F. Smith. Le Comité exécutif fut toujours formé de Van Horne, Angus et Smith et, à partir de 1906, de Chahoon Jr. Les assemblées des actionnaires ne faisaient qu'entériner les recommandations du C.A. et réélire celui-ci à l'unanimité chaque année sans que des conflits se manifestent. Le managing vice-president s'occupa de la liaison entre le C,A. et les officiers et, dès son entrée au Conseil d'administration, fut le promoteur de la majorité des initiatives provenant du Conseil.

\section{IV. - La Laurentide Company (I: 1911-1919)}

La réorganisation de 1911 consista en une réévaluation des actifs et en la création d'une nouvelle compagnie qui acheta l'ensemble des avoirs de la Laurentide Paper Co. et changea les actions de l'ancienne firme contre celles de la nouvelle à raison d'une action de la Laurentide Paper contre deux de la nouvelle Laurentide Company. La réévaluation des actifs se trouvait ainsi répartie proportionnellement parmi les actionnaires selon le nombre d'actions détenues et le gain de capital était alloué aux propriétaires en fonction de leur mise de fonds. Cette réorganisation était d'ailleurs attendue dans les milieux financiers. Les actions ordinaires de la Laurentide se cotaient sous leur valeur au pair en 1907, mais en 1908 elles se vendaient à 105 dollars, à 120 en 1909, à 140 en 1910 et atteignaient 220 dollars en 1911 avant la réorganisation. Les actions privilégiées étaient échangeables contre des actions ordinaires au même taux que celles-ci. Le capital-actions de la Compagnie passait avec la réorganisation de 3.6 millions à 7.2 millions de dollars et les actions privilégiées (dont seulement 2,000 restaient encore sur le marché en juin 1911) disparaissaient de la structure du capital de l'entreprise. Avec le rachat des obligations et les nouvelles émissions de 1910 et 1911 la proportion de capital-actions dans le passif augmentait à $80 \%$ en 1912. Durant la période cette gestion conservatrice de la Compagnie fut maintenue: en 1919 le capital-actions formait $61 \%$ du passif, et le surplus un 
$16.2 \%$ supplémentaire. Le remboursement des obligations fit que la dette fixe passa de $10 \%$ du passif en 1912 à $3.5 \%$ en 1919.

Des changements s'effectuent aussi dans la haute direction. En 1911 Charles F. Smith, membre du Conseil d'administration, meurt et il est remplacé par Frederick A. Sabbaton, qui conserva le poste jusqu'à la fusion de la Laurentide dans la Canada Power and Paper. Sabbaton était né aux États-Unis en 1871 ; polytechnicien, il avait immigré au Canada en 1902 et fut embauché comme "Assistant Manager» de la Laurentide la même année. Il n'était lié qu'à la Laurentide dont il était un cadre; plus tard il sera administrateur de la Laurentide Power, de la Canada Power and Paper et des autres compagnies reliées au groupe. À travers ce poste hiérarchique, Sabbaton acquit régulièrement des actions de la Laurentide pour devenir, à partir de 1911 un des principaux actionnaires de la firme.

En novembre 1913 James Ross, membre du C.A. de la Laurentide, mourut et fut remplacé par son fils, le commodore John K.L. Ross qui resta au C.A. jusqu'à la fusion de 1928. Le commodore Ross était en 1915 membre du Conseil d'administration du Canadien Pacifique. Il hérita des actions de son père - un des plus gros actionnaires - et il possédait 558 actions de 1915 à 1919. Le Conseil d'administration se composait alors de plusieurs des principaux actionnaires individuels: les 7 membres du C.A. en 1919 possédaient à leur nom 12,833 actions, soit $13.3 \%$ des actions souscrites; si on additionne les titres détenus par des membres de leur famille ils possédaient 16,474 actions, soit $17.2 \%$ du total. La séparation entre propriété et contrôle n'était donc pas très avancée au moment de la réorganisation de 1919.

En 1915 sir William Van Horne décédait à son tour et Charles R. Hosmer, administrateur, devenait vice-président, en remplacement de Chahoon Jr, élu président de la Laurentide. Sir Thomas Skinner fut désigné administrateur à la place de Hosmer. Skinner, noble anglais, actionnaire de la Banque de Montréal, du Canadien Pacifique (dont il était membre du C. A. depuis 1889) occupait le poste de président du Comité consultatif de la Banque de Montréal à Londres. Il resta au C.A. de la Laurentide jusqu'en 1923 et y retourna en 192425. Il comptait parmi les plus forts actionnaires de la Compagnie, possédant 500 actions de 1903 à $1911,1,333$ de 1911 à 1919 , et 3,999 de 1920 à 1928.

Si nous examinons maintenant la concentration de la propriété des actions, nous remarquons que tout au long de cette période une 
trentaine d'individus ou de sociétés détiennent environ $60 \%$ des actions donnant droit de vote dans la Compagnie. En 1919 il y avait cependant près de 700 actionnaires.

Parmi les autres principaux actionnaires, on compte John H.A. Acer. Né en Ontario en 1876, il est engagé en 1900 par la Laurentide dont il devient trésorier en 1905, et en 1906 à la fois trésorier et «general sales manager», postes qu'il conserva pendant 10 ans jusqu'à son départ pour la guerre européenne. De 1914 à 1916 il est le président de l'Association canadienne de pâtes et papiers. En 1917, il fonde une compagnie de ventes de papier à son compte.

Le secrétaire de la Compagnie, Ernest Alexander, un autre cadre, figure aussi dans la liste des principaux actionnaires. Il démissionne en juillet 1917 et vend ses actions. Les autres principaux actionnaires sont, comme dans la période précédente, des maisons de courtage de Montréal et quelques-unes de Toronto. Parmi les premières, la plus importante durant cette étape fut Charles Meredith and Co. (principal actionnairc institutionnel jusqu'en 1915); plus tard ce fut McDougall and Cowans de 1916 à 1919. D'autres maisons de courtage de Montréal se retrouvent dans la liste des propriétaires de plus de 500 actions: Burnett and Co., Craig and Luther, Th. Davidson and Co., L. Forget and Co., C. Simpson Garland and Co., Hanson and Ferguson, R. Moat and Co., Alex Patterson and Co., Oswals Bros. De Toronto la plus importante firme de courtiers qui reste actionnaire de la Laurentide est Osler and Hammond. Tout comme pendant l'étape antérieure, seulement un courtier, Edwin Hanson siège au C.A. de la Laurentide, ce qui montre le faible intérêt des courtiers au contrôle de la firme; ils n'y agissent que comme de purs investisseurs et le dividence semble être leur seul motif de détention des actions. Ils conservent d'ailleurs les mêmes titres pendant de longues années sans essayer de tirer profit des hausses et chutes des valeurs boursières.

La Compagnie est indépendante des banques à charte sur le plan du contrôle. Aucune banque n'est actionnaire important. La Compagnie emprunte, bien sûr, de la Banque de Montréal et concède ses opérations de «trustee» au Royal Trust (lié à la Banque de Montréal), mais encore ici les seuls liens de la Laurentide avec ces institutions financières proviennent de l'origine de ses directeurs (dont la plupart viennent du C.P.R. et de la Banque de Montréal) et de ses relations en tant que clients avec les dits banque et trust. 
En 1913 les États-Unis placèrent le papier journal parmi les produits d'entrée libre sur le marché américain; l'expansion des compagnies canadiennes et l'émigration vers le nord des firmes américaines s'intensifièrent. Alors qu'en 1913 le Canada avait produit 350,000 tonnes de papier journal contre 1,305,000 tonnes aux États-Unis, en 1919 le Canada produisit 795,000 tonnes et les États-Unis 1,375,000 ${ }^{19}$. La Laurentide ne fut pas étrangère à cette vague d'expansion papetière au Canada.

En 1911, la Compagnie élargissait la capacité de production de ses quatre principaux produits: la capacité de production de pâte mécanique passait de 150 tonnes par jour à 170 tonnes; en 1912, elle était portée à 200 tonnes, en 1913 à 230 tonnes et en 1918 à 280 tonnes. Dans la production de pâte au bisulphite, la capacité passait en 1911 de 70 à 80 tonnes par jour; en 1913, elle augmentait à 110 tonnes et en 1918 à 175 tonnes. Quant au papier journal, la capacité était portée de 150 à 170 tonnes en 1911 , à 200 tonnes en 1913 et à 210 en 1918. Enfin, la capacité de production de carton-pâte passait de 35 à 50 tonnes par jour durant cette période. La Laurentide restait la plus grosse entreprise canadienne de pâtes et papiers, mais de gros concurrents étaient déjà en train de lui enlever la première place: l'Abitibi Power and Paper et la Spanish River Pulp and Paper possédaient déjà une capacité de production de papier journal supérieure à celle de la Laurentide.

La Laurentide était donc une entreprise assez diversifiée dans son secteur. La production de pâte mécanique était presque entièrement consommée par l'usine de papier; le pourcentage de cette production vendue sur le marché ne dépassa $10 \%$ que vers les dernières années de la période; par contre la production de pâte au bisulphite s'est vendue dans une proportion croissante avec l'expansion de la production: de $25 \%$ en 1911 les ventes en prenaient plus de $50 \%$ en 1918-1919. La production de carton-pâte était résiduelle et resta presque stagnante.

Le moteur de cette expansion - au-delà de l'ouverture du marché américain et de l'embargo imposé au Québec par sir Lomer Gouin - fut la Première Guerre mondiale. De 1911 à 1915 les prix de vente du papier journal aux États-Unis se situaient entre 44 et 46 dollars; à partir de 1916 ils augmentèrent jusqu'à \$60. la tonne, et davantage par la suite. Le prix de vente de la pâte au bisulphite passait de 34 à 36 dollars entre 1911 et 1914 pour grimper à 85 dollars en

19 PPMC (le 3-2-1927): 149. 
1917 et retomber à $\$ 55$. la tonne en 1918-1919. Les coûts de production n'ayant pas augmenté dans la même proportion, les profits par tonne atteignaient des niveaux très élevés, en particulier pour la pâte au bisulphite.

Plusieurs éléments monopolistiques ont contribué à déterminer le niveau et la variation des prix et des profits. Le premier fut la formation d'un cartel destiné à faire monter les prix par la plupart des firmes américaines et canadiennes. L'enquête menée aux États-Unis par la Federal Trade Commission, sous la direction du commissaire Harris, à partir de juillet 1916, montra que la Newsprint Manufacturers Association (le nom du cartel) était une organisation illégale. Elle avait été constituée sans lettres patentes, sans articles d'association, sans règlements internes, ni procès-verbaux du Comité exécutif. Le but de cette association était de faire monter les prix du papier journal à un minimum de $\$ 60$. la tonne sur le marché nord-américain. Au Comité exécutif de la Newsprint Manufacturers Association citons le président de la Laurentide, Chahoon Jr, parmi d'autres figures représentatives de l'industrie canadienne et américaine. Les firmes représentées dans le cartel du côté américain étaient au nombre de quarante-trois (c'est-à-dire toutes sauf quatre en Amérique du Nord) y compris les plus importantes ${ }^{20}$. En novembre 1917 la N.M.A. fut légalement dissoute. Chahoon et G.H. Mead (président de la Spanish River Pulp and Paper Co.) écopèrent d'amendes de $\$ 2,500$. chacun pour avoir violé la loi Sherman ${ }^{21}$. Ce n'était pas une lourde peine pour la Laurentide qui avait réalisé un surprofit équivalant à l'amende chaque jour pendant un an et demi, grâce à sa participation au cartel. En septembre 1917, la F.T.C. avait imposé un prix maximum de \$50. la tonne aux achats gouvernementaux de papier journal. En novembre 1917 le prix maximum américain (pour le papier journal en rouleaux) était fixé à $\$ 60$., à $\$ 62$. en juin 1918 , à $\$ 70$. en septembre 1918, à \$75. en octobre 1918.

Entre-temps, au Canada, en octobre 1916, la Canadian Press Association demandait au gouvernement une enquête sur l'augmentation des prix du papier et sur les restrictions à l'offre du produit. En novembre, le Ministère des Finances mit sur pied une enquête, dirigée par $\mathbf{M}$. Pringle, et enjoignit les principaux producteurs de révéler leurs coûts de production et les prix de vente; en janvier 1917, Ottawa interdisait l'exportation de bois à pâte et en février, il fixait le prix maximum du papier journal en rouleaux à $\$ 50$. la tonne

\footnotetext{
20 PPMC (le 19-4-1917).

21 PPMC (le 29-11-1917).
} 
pour le marché interne canadien. Un an plus tard le prix maximum était fixé à $\$ 57$., mais les producteurs continuaient à se plaindre affirmant que leur coût de production avait augmenté à cause de la hausse des coûts de la matière première, de la main-d'œuvre et des frais d'entreposage.

Dans le cas de la pâte au bisulphite, aucune restriction n'était imposée et les prix grimpèrent rapidement, en particulier à partir de l'embargo suédois sur l'exportation de pâte chimique; cet embargo qui entra en vigueur en 1917 obligea la Grande-Bretagne et les ÉtatsUnis à s'approvisionner en Norvège et au Canada faisant ainsi monter davantage les prix. L'élimination de l'Allemagne et de l'Autriche des marchés mondiaux contribua aussi à la hausse des prix de la pâte.

Il faut souligner que la Laurentide sut profiter de la hausse vertigineuse du prix de la pâte au bisulphite en élargissant sa production dans ce domaine (comme on l'a vu) plutôt que dans celui du papier ou du carton. D'autre part, la Laurentide était traditionnellement liée au marché anglais et durant la guerre elle n'eut pas de difficulté à accéder à ce marché très rentable ${ }^{22}$.

D'après les données des enquêtes Pringle et Harris, la Laurentide possédait les coûts de production de papier journal les plus bas en Amérique du Nord en 1917. Le groupe de firmes choisies dans l'échantillon de la FTC établit ainsi les coûts pour 1917 du papier journal ${ }^{23}$ :

\begin{tabular}{|c|c|c|c|c|}
\hline Compagnie & $\begin{array}{c}\text { Coût } \\
\text { commercial } \\
\text { de } \\
\text { manufacture }\end{array}$ & $\begin{array}{c}\text { Dépenses } \\
\text { de } \\
\text { vente }\end{array}$ & $\begin{array}{l}\text { Dépenses } \\
\text { générales }\end{array}$ & Total \\
\hline $\begin{array}{l}\text { International P. Co. } \\
\text { Minnesota and Ont. }\end{array}$ & $\$ 43.14$ & $\$ 0.52$ & $\$ 1.66$ & $\$ 45.32$ \\
\hline P. Co. & 39.53 & - & 2.00 & 41.53 \\
\hline Belgo-Canadian & 38.83 & 0.95 & 2.71 & 42.49 \\
\hline Brompton & 50.77 & 1.29 & 1.42 & 53.48 \\
\hline Abitibi & 43.46 & 0.86 & 2.68 & 47.00 \\
\hline Laurentide Co. & 36.11 & 1.37 & 2.48 & 39.06 \\
\hline Price Bros. & 36.83 & 1.84 & 2.72 & 41.39 \\
\hline Donnacona & 43.36 & 1.20 & 1.51 & 46.07 \\
\hline Spanish River & 39.06 & 0.38 & 1.96 & 41.40 \\
\hline
\end{tabular}

22 PPMC (le 28-6-1917).

23 PPMC (le 13-6-1918). 
Les coûts de production de la Laurentide s'avéraient aussi, en 1917-1918, les plus bas dans la production de pâte mécanique et de pâte au bisulphite ${ }^{24}$. La tendance, cependant, était à une augmentation des coûts plus rapide que plusieurs de ses concurrents, dont la Belgo Pulp and Paper et Price Brothers.

La période 1911-1919 en fut une de grande diversification. En 1915 la Laurentide incorporait la Laurentide Power Company, une filiale destinée à la production d'énergie hydro-électrique pour les usines de pâtes et papiers. La Laurentide conserva $72 \%$ du capitalactions et le droit de nommer cinq des neuf membres du C.A. de la Laurentide Power; elle céda la partie minoritaire du contrôle de la filiale à la Shawinigan Water and Power Company qui prenait en charge le fonctionnement de l'entreprise. Ainsi, avec la séparation de ses usines hydro-électriques et la constitution de sa première filiale, la Laurentide s'assurait de l'approvisionnement d'énergie à bon marché. Le courant excédentaire était vendu à la Shawinigan. À sa fondation, la Laurentide Power avait une capacité de production de 125,000 chevaux vapeur et un actif de 10 millions de dollars.

Une deuxième filiale de la Laurentide fut la Canadian Export Paper Co. Cette société fut incorporée à Ottawa en août 1916. Elle était principalement (mais non exclusivement) vouée à l'exportation de papiers et produits connexes. Ses actionnaires étaient la Laurentide, Price Bros., la Belgo-Canadian et la Brompton; la proportion des actions détenues par chaque compagnie papetière variait en fonction de sa production. À sa fondation la Laurentide était le partenaire majoritaire avec 32.5\% des actions émises; Price Bros. et Belgo détenaient $29.3 \%$ chacune, et la Brompton le $8.9 \%$ restant. En 1918 Price devenait le premier producteur canadien de papier journal et recevait 33\% des actions de la C.E.P. Co., la Laurentide en conservait $31 \%$ et la Belgo $29 \%$. Cette compagnie de ventes était une nouvelle tentative de centralisation en vue d'augmenter les profits. Malgré son nom la Compagnie possédait un Département de ventes canadiennes, parallèlement à des Départements pour les ventes américaines et à l'étranger, ce qui prouve son intention de centraliser systématiquement les ventes de ces compagnies sur le marché du Dominion. Ce dernier but, toutefois, n'a pu être atteint lorsque la Commission Pringle fixa les prix du papier journal entre 1916 et 1919 . Et puisque la Commission Harris déterminait les prix sur le marché américain durant ces mêmes années, la C.E.P. ne put faire de profits que

24 PPMC (le 17-4-1919 et le 8-8-1918). 
sur les ventes outremer, qui ne signifiaient, en 1919 , que $25 \%$ de toute la production conjointe de papier journal des quatre firmes. Ainsi, en juin 1917 Chahoon déclarait à la Commission Pringle que la Laurentide vendait à Becker and Co. d'Angleterre, à travers la C.E.P. Co., 50 tonnes de papier journal par jour à un prix de $\$ 94$. et $\$ 100$. la tonne alors que sur le marché canadien il était fixé à \$50.

À l'intérieur de la Laurentide les opérations forestières se sont diversifiées et ont augmenté. Le Département forestier, créé en 1904, avait fait des relevés des limites de la Compagnie, étudié les types d'arbres et fourni le bois nécessaire à l'usine. À partir de 1908 la Compagnie commença les premières plantations sous l'initiative du chef du Département, Ellwood Wilson. En 1912 la Compagnie mit sur pied une pépinière à Grand-Mère et deux ans plus tard, elle envisageait le reboisement systématique comme moyen de renouveler sa matière première. Le reboisement passa de 300,000 arbres par année en 1915 à 1,000,000 d'arbres en 1919. La Compagnie fut la première aù Canada, et l'une des premières en Amérique du Nord, à mettre sur pied un programme aussi vaste de plantation d'arbres ${ }^{25}$.

Après une période de croissance lente (1903-1911), la Laurentide connut une étape de croissance rapide entre 1911 et 1919. L'actif total passa de 6.2 à 15.6 millions de dollars. La tendance à se financer par émission d'actions augmenta: si, en 1903-1911 la proportion moyenne du capital-actions dans le passif était de 36\%, en 1911-1919 cette proportion augmenta à $70 \%$; la capitalisation par obligations diminua régulièrement jusqu'à son élimination complète en 1919. Ces données montrent une gestion conservatrice décidée à réduire la dette fixe de la Compagnie. Elles montrent aussi que les profits très élevés de la guerre (obtenus sur les exportations et sur la vente de pâte chimique) étaient employés pour assainir financièrement la Compagnie, autant que pour le réinvestissement. En effet les dividendes versés furent maintenus bas, ce qui justifia en 1919 une réorganisation financière de la Compagnie.

\section{V. - La Laurentide Co. (1920-1928)}

Le 20 janvier 1920 la Laurentide recevait sa nouvelle charte. Le nom restait inchangé, mais la capitalisation était modifiée. Le capitalactions passait de 9.6 millions à 35 millions de dollars, dont 28.8 millions en actions étaient souscrits. En d'autres termes, les actifs

25 PPMC (le 15-1-1916), (le 15-8-1914); PM and WN (le 28-3-1925). 
étaient réévalués de façon à multiplier par trois leur valeur antérieure. Chaque actionnaire recevait trois actions de la nouvelle Compagnie par action qu'il détenait de l'ancienne. Les autres points de la charte restaient sans changements; la Compagnie recevait de vastes pouvoirs pour entreprendre toutes sortes d'activités économiques à l'exception des finances, des transports par chemin de fer et des assurances.

La cote boursière des actions de la Laurentide, qui était montée à $\$ 280$. en janvier 1920 après être restée au-dessus de $\$ 200$. en 1919 , retomba à sa valeur au pair aussitôt la réorganisation terminée.

La nouvelle charte ajoutait deux postes au Conseil d'administration. En juillet 1920 un poste fut rempli par sir Lomer Gouin, premier ministre de la Province du Québec de 1905 à 1920. Sous son gouvernement, en 1910, Québec avait décrété l'embargo sur l'exportation de bois à pâte coupé sur les terres de la Couronne. Cet embargo, comme nous l'avons souligné, fut un des principaux stimuli pour l'émigration vers le nord de l'industrie papetière américaine, et suscita la naissance des compagnies canadiennes. C'est ainsi que le Pulp and Paper Magazine of Canada pouvait se féliciter de la nomination de sir Lomer au C.A. de la Laurentide:

The attitude toward the Government of the Laurentide Co, with which Sir Lomer is now identified, has been always a generous one. The company naturally and properly desires to make a good profit.

/.../ By the association with the Laurentide Company Sir Lomer become definitely identified with Canada's great pulp and paper industry in a direct and personal way. Both may be truly congratulated $^{26}$.

De 1921 à 1925, sir Lomer devenait député fédéral pour Outremont et, de 1921 à 1924, ministre de la Justice à Ottawa, tout en conservant son poste d'administrateur de la Laurentide et d'actionnaire (400 actions) de la firme. Il devenait aussi, en 1920, membre du C.A. de la Banque de Montréal, poste qu'il conserva jusqu'en 1929, l'année de sa mort, où il venait d'être nommé lieutenant-gouverneur de la province de Québec.

L'autre poste fut attribué à sir George H. Montgomery. Né à Philippsburg, P. Q., en 1874, il avait étudié le droit à l'Université McGill et fut admis au Barreau en 1898. Il fut créé Chevalier en 1909. Il entra à la Montreal Light Heat, and Power Co. en 1905; par la suite, il

26 PPMC (15-7-1920): 742. 
devint administrateur des compagnies suivantes: Keystone Transportation Co., Brandram Henderson Ltd, Maguire Patterson and Palmer Co., Montreal Tramways Co., United Securities Co., Hydro-electric Bond and Share Corp., Investment Bond and Share Corp., Canadian Spool Cotton Co., Gatineau Power Co. Enfin il devint bâtonnier du Québec (1927), tout en conservant diverses fonctions à la tête de nombreuses firmes. Il œuvra dans l'industrie papetière du Canada à titre de conseiller général de la Canadian Pulp and Paper Association à compter de 1915, de directeur de la Canadian Export Paper Co. à compter de 1924, et plus tard de la Canadian International Paper, de la St-Maurice Valley Corporation et de la Canadian Newsprint Co. en 1927.

En septembre 1922 Richard B. Angus meurt et est remplacé au C.A. de la Laurentide par sir Vincent Meredith. Né à London, Ontario, en 1850, d'une famille d'origine irlandaise, fils d'un avocat de cette province, sir Vincent fit sa carrière à la Banque de Montréal. Il y entra en 1867 et entre 1910 et 1929 devint membre du Conseil d'administration, directeur général de 1911 à 1913, vice-président de 1912 à 1913, président de 1913 à 1927. En 1916 il était aussi administrateur du Canadien Pacifique et président du Royal Trust. En outre il était membre du Conseil d'administration de l'Université McGill, du Royal Exchange Assurance Co. of London, de The Guarantee Co. of North America, de la Consolidated Mining and Smelting Co. of Canada, de la Standard Life Assurance Co. of Edimburgh et d'autres firmes. Il était donc lié comme Gouin au groupe Banque de Montréal-Royal Trust-Canadien Pacifique.

Sir Thomas Skinner décède en juin 1926. John W. McConnell prend sa place au C.A. de la Laurentide. Né à Muskoka, Ontario, en 1877 il avait commencé très tôt sa carrière d'affaires à Toronto. En 1900 il déménagea à Montréal. En 1906 il était employé par la Banque de Montréal, dont il devint administrateur de 1925 à 1938. Il était aussi administrateur de la Montreal Light and Power Co., de Holt Renfrew Co. of Canada, de Dominion Rubber Co., de Brazilian Traction Light and Power, de Canada Steamships, de St. Lawrence Sugar Refineries et d'autres compagnies.

Les nouveaux administrateurs étaient aussi des actionnaires: Montgomery possédait 100 actions durant la période, Meredith 832, McConnell 100. Administrateurs de compagnies qui détenaient des parts dans la Laurentide: Royal Trust Co., St. Lawrence Sugar Refineries, entre autres, plusieurs, parmi eux étaient aussi administra- 
teurs de la Banque de Montréal, principal fournisseur de crédit à court terme de la Laurentide. Les maisons de courtage restent alors de très forts actionnaires (McDougall and Cowans en particulier). Sur la base des données dont nous disposons, il faut admettre un écart considérable entre propriété et contrôle au sein de la Laurentide: les Chahoon sont les principaux actionnaires, mais parmi les autres administrateurs, seuls Hosmer et Sabbaton sont des propriétaires d'un montant considérable d'actions. Gouin, McConnell, Montgomery et Hanson en possèdent peu à leur nom; Ross et Meredith sont des actionnaires moyens. Un autre indice de l'éparpillement relatif des actions est le nombre plus restreint des propriétaires de plus de 1,000 actions. En septembre 1924 la Laurentide comptait 2,318 actionnaires dont 2,078 au Canada, 164 aux États-Unis, et 45 en Angleterre. Cependant les administrateurs détenant moins d'actions remplissent des fonctions clefs, puisqu'ils assurent le lien avec le gouvernement québécois et canadien (Gouin, Montgomery) et avec d'autres firmes comme la Banque de Montréal et le Royal Trust (Meredith, McConnell, Gouin, Montgomery), tous en relations d'affaires avec la Laurentide.

Avec la réorganisation financière, la Laurentide recommençait son expansion. Encouragée par les prix d'après-guerre qui, contre toutes les attentes, restaient fermes, le C.A. décida en 1920 d'augmenter la capacité de production de pâte mécanique de 280 tonnes à 375 tonnes par jour et celle de papier journal de 210 à 350 tonnes et, en 1924, à 375 tonnes. La production de pâte au bisulphite restait inchangée ainsi que celle de carton-pâte. En 1923-24 la capacité de production des pâtes mécanique et au bisulphite et de carton-pâte fut légèrement réduite par la persistance des prix peu rémunérateurs dans ces produits.

Au début de la période les prix augmentaient sans cesse. En 1919 le prix f.o.b. à l'usine était de \$75. la tonne pour le papier journal standard en rouleaux. Dans le premier trimestre de 1920 il montait à $\$ 90 .$, au $4^{\mathrm{e}}$ trimestre à $\$ 130$. En 1921 ce fut la crise économique générale qui frappa aussi le secteur des pâtes et papiers. Au cours du 4 e trimestre de 1921 le prix était redescendu à $\$ 80$. et en 1922 , il tombait à $\$ 70$. En 1923 et 1924 il y eut une légère récupération et le prix remontait à $\$ 75$., mais à la deuxième moitié de 1924 il retombait de nouveau à \$73., en 1925 à \$70, en 1926 et 1927 à \$65.

Le mécanisme de fixation des prix était celui d'un marché oligopolistique: l'International Paper Company avait au début de la pé- 
riode une capacité de production de plus de 1,000 tonnes par jour. La Canadian Export Paper mettait en marché la production combinée de trois entreprises du Québec (Laurentide, Price et Brompton) pour un total de quelque 700 tonnes par jour en 1920 et près de 1,200 en 1927. La George H. Mead Co., compagnie de ventes de l'Abitibi et de la Spanish River, mettait en marché 900 tonnes en 1920. Ces trois grands vendeurs déterminaient le prix sans qu'il y eût un leadership de la part de l'un ou de l'autre. Les prix se fixaient lors de la signature des contrats de vente pour l'année suivante (de septembre à novembre) mais après l'accord sur les conditions de vente, les compagnies papetières ou leurs agents pouvaient effectuer des réductions de prix ou des remboursements de frais de transport, et faire ainsi descendre les prix fixés initialement.

La Laurentide ne pouvait que suivre ces mouvements de prix à la baisse. Une première solution fut la recherche de moindres coûts. Il ne fait pas de doute que la Compagnie réussit à réduire les coûts de sa production principale. Une autre réponse fut la recherche de prix outremer plus rémunérateurs, à travers la Canadian Export Paper Co. Nous reviendrons sur ce point en parlant des compagnies de vente.

La cause principale de la chute des prix était le surinvestissement. La production américaine de papier avait atteint un record en $1920(1,512,000$ tonnes $)$, niveau qu'elle ne dépassera qu'en 1925 à cause de la crise de 1921-22. Après une année de croissance, 1926, la production américaine retombait en 1927 à un niveau inférieur à celui de 1920 (1,485,000 tonnes). La production canadienne par contre se développait sans cesse. En 1920 elle atteignait 876,000 tonnes; après une légère chute en 1921 elle remontait à $1,082,000$ tonnes en 1922. En 1926 elle dépassait pour la première fois la production-américaine, avec 1,882,000 tonnes. En 1927 elle était de 2,087,000 tonnes: en sept ans elle avait plus que doublé son niveau. Le Canada était devenu en 1926 le premier producteur mondial de pâtes et papiers fournissant $25 \%$ de la production mondiale. Même la chute des prix n'arrêtait pas ce mouvement: en 1925 dix nouvelles machines à papier furent installées au Canada (dont 2 pour Price Bros. et 2 pour Wayagamack News); en 1926 quatorze nouvelles machines furent mises en fonctionnement (dont 4 pour C.I.P., 2 pour Port Alfred Pulp and Paper, 2 pour Powell River et 2 pour la St. Lawrence); en 1927 dixhuit nouvelles machines étaient mises en fonctionnement (dont 4 autres pour Canadian International Paper, 2 pour Ste. Anne, 2 pour Manitoba et 2 pour l'Anglo-Canadian). De 1919 à 1927, 82 nouvelles 
machines à papier furent installées en Amérique du Nord, dont 63 au Canada, 14 aux États-Unis et 5 à Terre-Neuve. Et pourtant quelques voix isolées s'étaient fait entendre pour dénoncer ce surinvestissement, entre autres, celle d'Hubert Biermans, président de la BelgoCanadian et administrateur de la Canadian Export Paper Co., qui prévoyait en 1924 une chute vertigineuse des prix et un crash dans l'industrie. Et puisqu'il croyait à ses déclarations, il vendit en 1925 sa compagnie à la St. Maurice Paper Co., commençant ainsi la vague de fusions qui se continua durant plusieurs années. Mais l'opinion était majoritairement contraire à celle de Biermans. Aussi tard qu'en mai 1926, le Pulp and Paper Magazine of Canada, l'organe des manufacturiers canadiens, attaque le «overproduction bugbear» 27 et affirme que l'industrie papetière du Canada a devant elle des années brillantes. Un an plus tard, devant la chute des prix et les mesures prises par les manufacturiers pour y faire face, la revue affirme que «l'expansion est venue trop rapidement» sans attendre la croissance de la demande nord-américaine ${ }^{28}$.

La Laurentide subit la vague de surinvestissement mais elle n'y participa pas. Sa croissance s'est arrêtée en 1920-22 avec l'incorporation des machines nos 8 et 9 pour la production de papier journal. Ces machines atteignirent leur pleine capacité de production en 1924 et, à partir de cette date, aucun nouvel équipement ne fut incorporé aux moulins. La Compagnie s'est contentée d'améliorer sa production forestière par l'utilisation (la première en Amérique du Nord) d'avions pour l'étude des bois, d'accélérer le reboisement (à la fin de la période elle produisait 6 millions d'arbres par an) et d'élargir ses limites. La politique conservatrice de son Conseil d'administration l'empêcha sans doute de participer à la vague de surinvestissement qui provoquera les faillites de 1928-31.

La réorganisation de 1920 marqua la dernière capitalisation de la Laurentide. Le capital-actions ayant été "mouillé », il passait de $\$ 9,600,000$. à 28.8 millions de dollars par simple revalorisation des actifs. Les profits cependant ont conservé les niveaux de 1919, ce qui diminua le taux de rentabilité financière de $19 \%$ en 1919 à 6-7\% en 1920-27. Les dividendes tombèrent à $6 \%$ pour toute la période et la cote boursière des actions retomba autour de sa valeur nominale au pair $(\$ 100$.$) .$

27 PPMC (le 25-5-1926).

28 PPMC (le 26-5-1927). 
Du côté du passif, en 1920, les dernières obligations furent complètement rachetées des mains du public et en 1926 le prêt de la Banque de Montréal qui avait servi à financer l'expansion de 1920-22 fut entièrement payé. À partir de 1924 plus de $80 \%$ de la capitalisation de la firme était formée du capital-actions. De ce point de vue, la Laurentide poursuivit pendant cette dernière étape sa politique traditionnelle d'indépendance vis-à-vis des institutions financières en général et des banques à charte en particulier.

L'organisation de la Laurentide correspond encore, à la fin de la période, à la structure départementale qui est celle décrite par Chandler. Le graphique no 1 tente d'illustrer cette structure. On y a enlevé les assistants et le personnel subalterne pour rendre moins lourd le tableau.

À remarquer trois groupes de départements: le groupe relié aux opérations forestières (reboisement et coupe du bois), le groupe relié à la production de pâte, carton et papier avec ses départements accessoires, et les départements de secrétariat (s'occupant des livres) et la trésorerie (s'occupant des finances). L'absence d'un département des ventes est due à l'existence de la Canadian Export Paper Co. Aux côtés du Conseil d'administration on remarque le Comité exécutif, dont l'existence s'avérait plutôt légale que réelle puisqu'il ne se réunissait que sporadiquement et le Conseil de la Laurentide, un organe consultatif formé de représentants ouvriers (délégués par département) et du Conseil d'administration. Tous les projets passaient par Chahoon ou provenaient de lui; il réglait à la fois les questions financières et les détails techniques. Très souvent, les réunions du C.A. de la Laurentide se réduisaient à approuver les plans de Chahoon $\mathrm{Jr}$ ou à lui donner pleins pouvoirs pour envisager une expansion, une prise de participation, etc.

\section{De la Canadian Export Paper Co. à la Canadian Newsprint Co.}

En 1921, la Belgo-Canadian se retirait de la CEP pour former sa propre compagnie de ventes. Durant la même année, la St. Maurice Paper rompait ses accords avec la CEP et celle-ci resta comme agence de ventes pour la Laurentide, la Price Bros et la Brompton. Entretemps l'expansion de la Price faisait que cette firme devenait l'actionnaire principal de la CEP: à partir de 1923 Price Bros. détenait 1,626 actions de la CEP contre 1,596 pour la Laurentide et 249 pour la Brompton. 
GRAPHIQUE $\mathrm{N}^{\circ} 1$

Laurentide Company: structure administrative (1927)

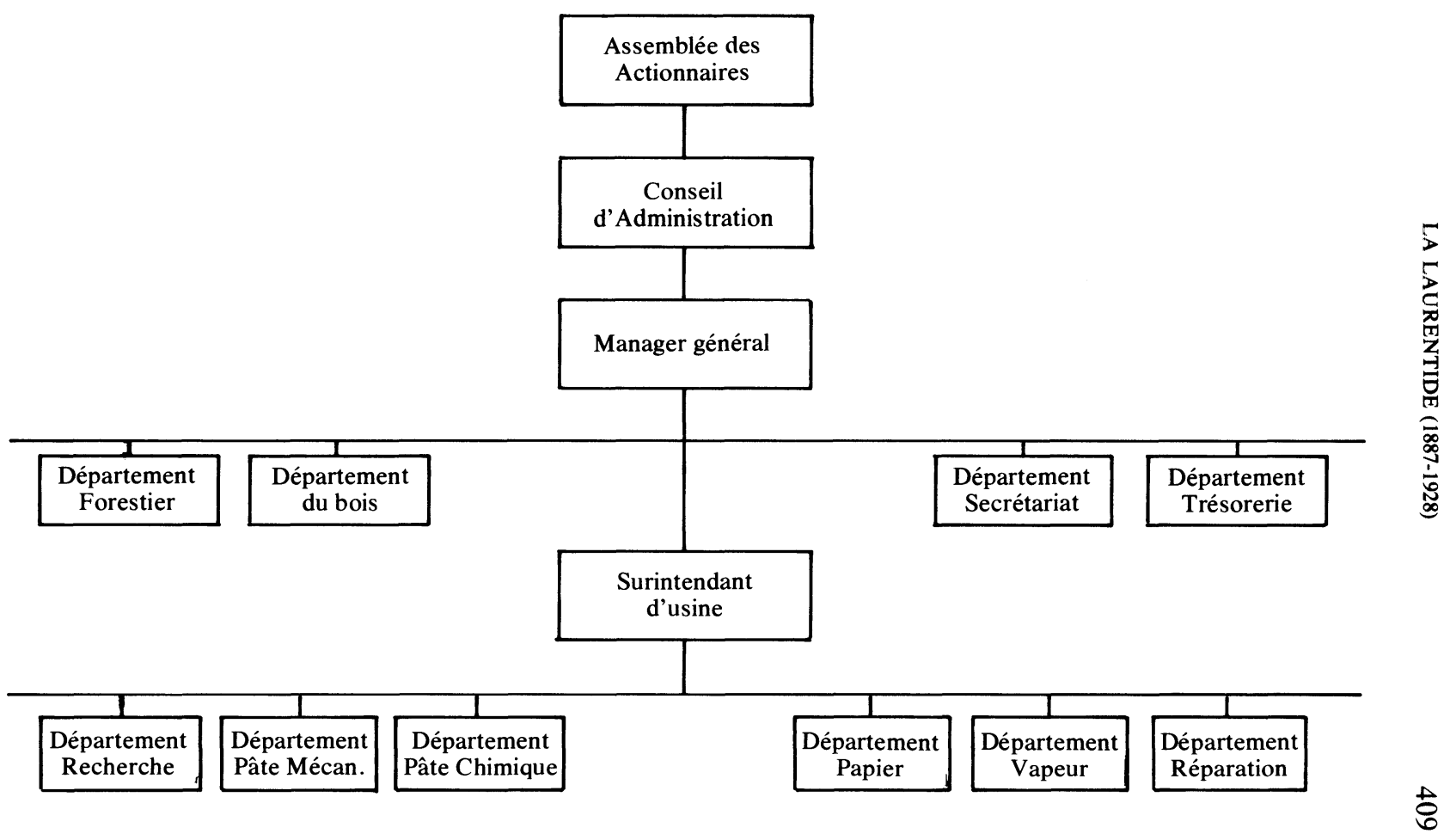


Les présidents des trois compagnies membres formaient le noyau du C.A. de la Canadian Export Paper. En octobre 1924 sir William Price mourut et fut remplacé à la tête des compagnies du groupe (et à la CEP) par son fils, John H. Price. Le «management» était fourni par George Steele, general manager jusqu'en 1921. À cette date il démissionna et fut remplacé par un board of management formé de trois experts: E.L. Crooker (de la Laurentide), F.R. Thorn (de Price Bros.) et R.H. Bryan (de la Brompton). En 1923 on revint au système du manager individuel, poste qui sera occupé par Crooker avec un comité consultatif sur lequel siégeaient Thorn et Bryan.

Dans l'ensemble la CEP semble avoir été une organisation de ventes très efficace. Le tarif préférentiel australien (aboli seulement en 1925) favorisait l'importation de papier en provenance du Royaume-Uni et réduisait à presque rien les exportations canadiennes vers ce marché. La concurrence scandinave et anglaise empêchait la CEP de pénétrer dans d'autres marchés comme l'Afrique du sud, l'Amérique latine et l'Inde. Dans ces conditions, de $70 \%$ à $80 \%$ des ventes de la CEP se faisaient aux États-Unis, $10 \%$ au Canada et le restant sur les marchés outremer. Plusieurs des grands journaux américains (le $\mathrm{New}$ York Times, la chaîne Hearst) comptaient parmi les clients les plus fermes de la CEP, qui réussit à placer la presque totalité de la production des compagnies membres. En 1921 la Canadian Export Paper s'était spécialisée dans la vente du papier journal et elle abolissait son département de papiers spéciaux.

Cependant la chute des prix obligera les compagnies canadiennes à se regrouper dans une compagnie de ventes commune pour maintenir les prix. En mai 1927 la Canadian Export Paper Co., la George H. Mead Co. et la St. Maurice Valley Sales se fusionnaient pour former la Canadian Newsprint Company. La nouvelle agence de ventes, avec une capitalisation de 5 millions de dollars en actions privilégiées et 10,000 actions sans valeur au pair, réunissait la production de papier journal de onze compagnies canadiennes: La Laurentide Co., la Price Bros., la Port Alfred Pulp and Paper Corp., la St. Maurice Valley Corp., la Canada Paper Co., la Ste. Anne Paper Co., la Spanish River Pulp and Paper Mills, la Fort Williams Paper Co., l'Abitibi Power and Paper Co., la Manitoba Paper Co. et l'AngloCanadian Pulp and Paper Co. La production annuelle totale estimée de ces compagnies s'élevait à 1,500,000 tonnes de papier journal, soit les $3 / 4$ de la production canadienne. L'objet de la fusion était l'ouverture des marchés externes, la réduction des coûts de vente et de transport et surtout le maintien des prix sur le marché nord amé- 
ricain. Le Conseil d'administration de la nouvelle Compagnie était formé de George Chahoon, sir Herbert Holt, George H. Mead, Alexander Smith, John H. Price, J.H. Gundy, George H. Montgomery, Frank W. Clarke et W.N. Hurlbut. Dans cette gigantesque compagnie de ventes, la Laurentide détenait $8 \%$ des actions; elle nomma le premier vice-président, E.L. Crooker, en plus de deux administrateurs (Chahoon et Montgomery).

Les autres' filiales de la Laurentide (la Laurentide Power en particulier) continuèrent leurs opérations sous son contrôle. La Laurentide en acquit même de nouvelles dont la Laurentide Ottawa, formée de la fusion de deux compagnies forestières achetées dans la région de l'Outaouais.

\section{La fusion}

La chute des prix provoqua non seulement le regroupement des compagnies de ventes mais aussi la fusion des firmes papetières ellesmêmes. La vague de fusions commença en 1925 quand la St. Maurice Paper absorba la Belgo, initiative de sir Herbert Holt. La nouvelle compagnie, la St. Maurice Valley Corporation avait une capacité de 1,000 tonnes de papier journal par jour ou 300,000 par an. En octobre 1927 l'Abitibi acheta la Spanish River, la Fort Williams Power, la Manitoba Paper Co., la Ste. Anne Paper Co. et la Murray Bay Paper Co. La Compagnie ainsi créée, avec un actif de 178 millions de dollars et une capacité de production de 650,000 tonnes par an, devint la plus grande firme industrielle au Canada ${ }^{29}$. À ce moment-là il ne restait que quelques compagnies papetières indépendantes au-delà des trois grands groupes (l'International Paper, le St. Maurice Valley et l'Abitibi-Spanish River). Ces compagnies indépendantes étaient la Price, la Laurentide, la Wayagamack, la Brompton, la St. Regis et quelques autres de moindre importance. Au milieu de janvier, on annonçait l'absorption de Donnacona Paper Mills par Price Bros. et la fusion de la Laurentide Company dans le groupe St. Maurice Valley dirigé par Holt. La proposition du Conseil d'administration de la Laurentide aux actionnaires se lisait ainsi :

At a meeting of the board held this afternoon your directors, after conferring with directors of the St. Maurice Valley Corp. approved a plan for the amalgation of the common stock in-

29 PPMC (le 27-10-1927). 


\begin{abstract}
terests of your compagny and of $S t$. Maurice l'alley Corporation. The plan involves the exchange of shares of Laurentide Co. Ltd, for shares and debentures of a new company to be incorporated under the name of Canada Power and Paper Corporation or some other suitable name, and to be organized upon the following lines:

The new company will be a Quebec corporation with its head office at Montreal. Its authorized share capital will consist of 750,000 shares without nominal or par value. Shares will be alloted and issued to holders of shares of Laurentide Co. Ltd. on the basis of one share of the new company's stock for each share of Laurentide Co. Ltd. transferred to the new Company. In addition, holders of Laurentide Co. Ltd will be entitled to receive upon such tranfer $\$ 100$. principal amount of $5 \frac{1}{2}$ per cent thirty year sinking fund gold debentures of the new company for each share transferred ${ }^{30}$.
\end{abstract}

Pour leur part, les actionnaires de la St. Maurice Valley recevraient $2 \frac{1}{2}$ actions ordinaires sans valeur au pair pour chaque action transférée de la St. Maurice. La nouvelle Compagnie aurait 688,000 actions sans valeur au pair émises (sur les 750,000 autorisées) et des débentures pour 28.8 millions de dollars. Les moulins ainsi unifiés auraient une capacité quotidienne de 1,400 tonnes de papier journal, soit 520,000 tonnes par année. La nouvelle Compagnie détiendrait les filiales de la Laurentide Company. À la tête du Conseil d'administration de la Canada Power and Paper, on retrouve celui qui avait eu l'initiative de la fusion, sir Herbert Holt, président de la Banque Royale du Canada, les deux vice-présidents, George Chahoon et George McKee. Ce dernier était aussi «managing director» de la St. Maurice Valley Corp. En février 1928 l'offre fut acceptée par les actionnaires des deux compagnies et la Laurentide disparut comme entité économique indépendante bien qu'elle conserva sa charte jusqu'en 1949, année de la dissolution légale de la Compagnie.

\title{
VI. - Conclusions et quelques hypothèses exploratoires
}

Dans ce dernier chapitre, il s'agit de tirer quelques conclusions globales et d'énoncer un certain nombre d'hypothèses exploratoires

$30 \quad$ Procès-verbaux du C.A., livre no 7: 182. 
de caractère sociologique sur les caractéristiques de la bourgeoisie montréalaise, telle que représentée à la Laurentide, et de son prolétariat.

Les données sur la distribution de la propriété des actions nous permettent d'affirmer une tendance certaine vers l'éparpillement des titres. La Compagnie avait cinq actionnaires à sa fondation, plusieurs centaines en 1903 et près de 3,000 à sa fusion en 1928 . Et pourtant le nombre d'actionnaires qui détiennent plus de $50 \%$ des actions n'augmente pas de façon significative: en 1887 quatorze actionnaires (tous des particuliers) possédaient $85 \%$ des actions; en 1903, vingt actionnaires en détenaient 65\% ; en 1927 cinquante actionnaires (si on enlève les administrateurs ayant moins de 1,000 actions) possédaient 55\% du capital-actions souscrit. Par contre le Conseil d'administration détient de moins en moins d'actions à son nom. En 1887 les cinq administrateurs possédaient à leur nom 50\% des actions; en 1903 les 7 membres du C.A. en détenaient (en leur nom seulement) $38 \%$; en 1927 ils n'en détenaient que $8 \%$. Le contrôle familial cependant est important. Chahoon n'a à son nom que quelques actions, mais sa famille - sa femme Élizabeth, son père et son fils - possèdent $3.3 \%$ des actions de la firme qui sont déposées au Royal Trust, comme l'indique le registre de Transfert d'actions. Les Hosmer, les Angus, les Van Horne restent des actionnaires importants. Le fait le plus remarquable à partir de 1911, est la croissance des investisseurs institutionnels, et en particulier, des maisons de courtage. Un seul courtier cependant se retrouve au C.A. de la Laurentide: Edwin Hanson. Aucune des principales maisons de courtage actionnaires (McDougall and McDougall, McDougall and Cowans, etc.) n'est représentée à la direction de la firme. Ceci peut indiquer soit le désintéressement des courtiers pour le contrôle, soit le désintéressement des propriétaires représentés par les courtiers, soit encore que plusieurs des administrateurs représentent des intérêts qui se retrouvent derrière les maisons de courtage. Nos données ne nous permettent pas d'accepter ou de rejeter en bloc la théorie de Berle et de Means. L'éparpillement des actions est certain mais la majorité reste concentrée dans les mains d'au plus une cinquantaine d'actionnaires. Le C.A. détient de moins en moins d'actions, mais les familles sont encore très puissantes. Et l'on ignore encore le rapport entre la propriété des maisons de courtage et le contrôle de la corporation.

Beaucoup de données tendent à réfuter la théorie du capital financier de Hilferding. Aucune banque à charte ne détient d'actions de la Laurentide en quantité autre que négligeable. Les quelques socié- 
tés de fiducie représentées dans la liste des actionnaires détiennent des actions pour le compte de tierces personnes (pour la famille Chahoon par exemple). Les prêts bancaires, presque tous de la Banque de Montréal depuis 1900 et de la Banque de Commerce précédemment, étaient à court terme et la Compagnie s'est empressée de les rembourser à chaque occasion. À plusieurs reprises on parle de l'importance de constituer un fonds de roulement interne et de se rendre totalement indépendants des banques. Il est vrai que plusieurs directeurs de la Banque de Montréal (et du Royal Trust) se trouvent au C.A. de la Laurentide. Mais ils le font autant à titre d'actionnaires individuels qu'en tant que représentants de l'institution créancière, sans que ceci implique un contrôle d'aucune sorte. Pour résumer, on peut dire que la Laurentide appartient à un «groupe financier» ou à un groupement d'intérêts (celui de la Banque de Montréal, du Royal Trust et du CPR) mais elle n'est nullement soumise au «capital financier», c'est-à-dire au capital bancaire, selon la thèse de Hilferding.

La théorie de Chandler, par contre, semble, sous plusieurs aspects, confirmée. À partir de l'adoption en 1893 d'une structure départementale, l'organisation ne cessa de se développer dans ce sens. De nouveaux départements furent ajoutés mais la structure administrative resta la même. Une caractéristique particulière de la Laurentide est la forte centralisation autour d'une seule personne: G. Chahoon Jr, "general manager» et vice-président de 1902 à 1915; «manager» général et président de 1915 à 1928. Toute l'expansion, la diversification, l'achat de terrains, le choix de techniques, etc, sont décidés par ce manager professionnel, devenu principal propriétaire de la firme au cours des 25 ans où il fut à sa tête. Cette centralisation amoindrit les pouvoirs du Comité exécutif. Quant au Comité financier il n'exista que pendant quelque temps, lors des crises de croissance des premières années sous la présidence de Pagenstecher.

La bourgeoisie, représentée à la Laurentide, est issue du secteur financier et des chemins de fer. C'est dans le transport, le commerce et les finances que l'accumulation s'est faite principalement au Canada au cours du XIXe siècle. Cette thèse de T. Naylor ${ }^{31}$ semble être confirmée par notre étude. Si nous nous demandons quelle est l'origine des capitaux canadiens qui achètent la Laurentide des intérêts 2 vol.

31 T. Naylor, The History of Canadian Business 1867-1914 (Toronto, 1975), 
américains la réponse est claire: le Canadien Pacifique, la Banque de Montréal et des entreprises financières, commerciales, etc., qui y étaient associées. En 1903, des 7 membres du C.A., trois étaient aussi administrateurs de la Banque de Montréal (Angus, Hosmer et Ross), trois étaient administrateurs du CPR (Angus, Hosmer et Van Horne). En 1911 nous trouvons un seul changement: le général Alger a été remplacé par Sabbaton, un manager professionnel, qui accumula des actions au cours des 20 ans où il siégea au C.A. de La Laurentide. À sa mort en 1915 Van Horne fut remplacé par sir Thomas Skinner, de la Banque de Montréal. Et en 1927 parmi les 9 membres du C.A., 4 (Gouin, Meredith, Mc Connell, Hosmer) faisaient partie des administrateurs de la Banque de Montréal, et deux (J.K.L. Ross et Meredith) du Canadien Pacifique. Les autres liens des administrateurs couvrent des dizaines d'autres firmes sans beaucoup de recoupements. Un dernier fait à signaler: aucun des administrateurs de la Laurentide ne se trouve au C.A. d'une banque autre que la Banque de Montréal. Il y a ici une indication intéressante sur la structure des «regroupements d'intérêts» au sein de la bourgeoisie montréalaise.

Une dernière remarque concerne la nationalité des administrateurs et la nationalité de la firme. Plusieurs des plus importants administrateurs sont nés aux États-Unis et conservaient même la nationalité américaine (Pagenstecher, Curtis, Van Horne, Chahoon, Sabbaton, Alger); plusieurs autres étaient nés en Angleterre (Angus, Meredith, Skinner, Hanson, Ross). La majorité cependant était canadienne, et la firme se range clairement du côté de l'industrie canadienne lors des divers conflits contre les manufacturiers américains: l'embargo sur le bois à pâte de 1910, la menace tarifaire de la résolution Underwood en 1920. Elle s'y range aussi et surtout lors de la fusion de 1928, en rejoignant le seul groupe majoritairement canadien: celui de sir Herbert Holt et de la St. Maurice Valley Corporation. 